\title{
The Total Deformation and Its Role in Heavy Precipitation Events Associated with Deformation-dominant Flow Patterns
}

\author{
GAO Shouting $^{1^{*}}$ (高守亭), YANG Shuai ${ }^{1,2}$ (杨帅), and XUE Ming ${ }^{3}$ (薛明) \\ ${ }^{1}$ Laboratory of Cloud-Precipitation Physics and Severe Storms (LACS) \\ Institute of Atmospheric Physics \\ Chinese Academy of Sciences \\ Beijing, 100029, China \\ ${ }^{2}$ Graduate University of Chinese Academy of Sciences, Beijing, 100049, China \\ ${ }^{3}$ School of Meteorology and Center for Analysis and Prediction of Storms \\ University of Oklahoma, Norman Oklahoma, USA
}

\begin{abstract}
In this paper, it is elucidated that the total deformation (TD), defined as the square root of the sum of squared stretching deformation and squared shearing deformation, is an invariant independent of the coordinate system used. An idealized flow field is then constructed to demonstrate the confluence effect of a non-divergent and irrotational deformation field on moisture transport. To explore the characteristics and role of TD, two heavy rainfall cases associated with a front with shear-line, are analyzed using the WRF model output data. One of the cases occurred in the middle and lower reaches of the Yangtze River (MRYR) over China and the other occurred in north China. It is found that right before the occurrence of precipitation, the effect of the confluence induced by deformation on moisture transport provides a favorable condition for precipitation.

During the precipitation, both location and orientation of the zone of large TD coincide with the confluent shear line. The rainbands are nearly parallel with, and located lightly to the south of the zones of large TD and the confluent shear line. The TD in the lower troposphere increases in value as precipitation persists. When TD approaches its maximal value, the next 6hour precipitation reaches its peak correspondingly.

A tendency equation for TD is derived. The analysis of linear correlation and RMS difference between individual terms in the total deformation equation and the sum of the terms shows that the pressure gradient plays a major role in determining the local change of total deformation.
\end{abstract}

Key words: deformation, confluence, precipitation, equation

\section{Introduction}

Among the basic variables that describe the atmosphere, namely, temperature, pressure, humidity and wind velocity, only the wind velocity is a vector. At the mesoscale where the

*E-mail: gst@lasg.iap.ac.cn 
characteristic scale is less than the Rossby radius of deformation, it is known that the wind field determines the mass field through geostrophic adjustment (Rossby, 1937, 1938; Yeh, 1957; Yeh and Li, 1982; Zeng, 1963a,b,c). At the even smaller, convective storm scale, the importance of wind field is even more evident. It is known that the environmental wind profile has a strong control on the storm types (Weisman and Klemp, 1982) and storm dynamics (e.g., Walter and Thorpe, 1979). The environmental helicity, defined as the dot product of the environmental velocity and vorticity vectors, plays an important role in the storm longevity and the rotation characteristics of thunderstorms (e.g., Lilly, 1986a,b; Etling, 1985; Davies-Jones, 1984; Droegemeier et al., 1993; Lu and Gao, 2003).

The importance of the wind field has been recognized by many researchers. Pettersen (1956) showed that a 2D linear wind field could be expressed as a combination of translation, divergence, deformation, and vorticity components. Other researchers, including Wiin-Nielsen (1973) and Norbury (2002), have also discussed the above properties of wind fields in some details. Traditionally, the divergence and vorticity are two quantities that have received most attention. The prognostic equations for vorticity and divergence derived from the equations of motion have been used extensively in the literature as well as in textbooks for studying dynamics of fluid flows and various atmospheric phenomena (Wu and Tan, 1989). The deformation has, in comparison, received much less attention except in studies of frontogenesis. For certain types of precipitation systems, such as those associated with the eastern China Meiyu front, the largescale flow pattern often exhibits a dominant deformation pattern at the low levels while the divergence and vorticity are sometimes of smaller magnitudes. For these types of systems, it is important to understand the role of deformation, in the triggering and maintenance of the precipitation, and to gain insight on the time evolution of the deformation field.

In section 2 of this paper, an idealized flow model is first constructed to demonstrate the confluence effect of non-divergent and irrotational deformation on moisture. In section 3, it is shown that the total deformation is an invariant independent of the coordinate system used. A prognostic equation of the total horizontal deformation is derived. In section 4, two heavy rainfall cases, one associated with a Meiyu front in nearly east-west orientation over eastern China, and the other associated with a front in nearly north-south orientation over northern China, are numerical simulated and analyzed. In section 5, the deformation fields and their roles associated with these two heavy rainfall cases are diagnosed and analyzed using the WRF model output data. In section 6, the relative contributions to total deformation of the terms in total deformation equation are analyzed for the two rainfall cases. Conclusions are given in section 7.

\section{An idealized flow field model}

A pure 2D deformation flow is non-divergent and irrotational. For this reason, it by itself does not produce vertical lifting at the low levels or create convergence through the Ekman pumping effect. But deformation is important in frontogenesis. It increases horizontal temperature gradient therefore baroclinicity through advection, which also leads to frontogenesis. In addition, the deformation flow can play an important role in advecting moisture into a precipitation region.

To illustrate the role of a deformation-dominated flow field that is typical of the flow pattern associated with the Meiyu front, we construct an idealized flow field of the typical 'saddle' pattern. We construct our idealized flow field, through a linear combination of two functions. Function 1 defines a vortex (Luther White, personal communication, 2006) 


$$
V_{T}(R)=V_{0}\left(\frac{\Phi_{1}+\Phi_{2}}{2}\right)
$$

where $\Phi_{n}\left(R, R_{0}\right)=\frac{2 n R_{0}^{2 n-1} R}{(2 n-1) R_{0}^{2 n}+R^{2 n}}, \quad n=1,2, \ldots \ldots$

This vortex contains two free parameters, $n$ and $R_{0}$, where $n$ is an integer, $R_{0}$ is a characteristic radius, and $R$ the radius from the vortex center. This vortex looks like a smoothed version of the Rankine combined vortex (Harasti and List, 2005); in particular, it avoids the singularity point associated with the Rankine combined vortex at $R=R_{0}$ (Fig. 1).

Note that, here $V_{T}$ is the tangential wind speed with the vortex, $V_{0}$ the maximum value of $V_{T}$ at radius $R_{0}$, and $R$ is the radius for the constructed high and low circulations.

And function 2 specifies a pure deformation flow:

$$
\left\{\begin{array}{l}
u=b x \\
v=-b y
\end{array}\right.
$$

where $\mathrm{b}$ is a positive constant. A linear combination of them gives

$$
\left(\begin{array}{l}
u \\
v
\end{array}\right)=\alpha K(m) V_{T}(R)\left(\begin{array}{l}
-\sin \theta \\
\cos \theta
\end{array}\right)+(1-\alpha)\left(\begin{array}{l}
b x \\
-b y
\end{array}\right) .
$$

where $\quad K(m)=\left\{\begin{array}{ll}1, & \text { when } m=1 \text { or } 3 \\ -1, & \text { when } m=2 \text { or } 4\end{array}\right.$, (where $m=1,2,3,4$ are denote the No.m quadrant); $\tan \theta=\frac{y-Y_{0 m}}{x-X_{0 m}}, \sin \theta=\frac{y-Y_{0 m}}{R}, \cos \theta=\frac{x-X_{0 m}}{R}, x=(i-51) \Delta x, y=(j-51) \Delta y$, (where the total grid points are $101 \times 101), R=R(x, y)=\sqrt{\left(x-X_{0 m}\right)^{2}+\left(y-Y_{0 m}\right)^{2}}$.

$$
\left(X_{0 m}, Y_{0 m}\right)=\left\{\begin{array}{ll}
\left(X_{01}, Y_{01}\right)=(25 \Delta x, 25 \Delta y) & \text { when } m=1 \\
\left(X_{02}, Y_{02}\right)=(-25 \Delta x, 25 \Delta y) & \text { when } m=2 \\
\left(X_{03}, Y_{03}\right)=(-25 \Delta x,-25 \Delta y) & \text { when } m=3 \\
\left(X_{04}, Y_{04}\right)=(25 \Delta x,-25 \Delta y) & \text { when } m=4
\end{array}\right. \text { are the four centers of constructed }
$$

high and low circulations. Deformation coefficient $b=1.6 \times 10^{-5}$. The center of flow region is at the origin of the coordinate system. We calculate the velocity on a $101 \times 101$ grid at a grid spacing of $60 \mathrm{~km}$ (i.e., we take $\Delta x=\Delta y=60 \mathrm{~km}$.). In Eq. (1), $\alpha$ is a weight determined by

$$
\alpha=\left\{\begin{array}{cc}
0, & \mathrm{R}_{\mathrm{i}, \mathrm{j}}>\mathrm{R}_{0} \\
\frac{R_{i, j}}{25 \Delta x}, & \mathrm{R}_{1}<\mathrm{R}_{\mathrm{i}, \mathrm{j}} \leq \mathrm{R}_{0} \\
1, & 0<\mathrm{R}_{\mathrm{i}, \mathrm{j}} \leq \mathrm{R}_{1}
\end{array}\right.
$$


where $\Delta x$ is the grid spacing. $R_{0}=1200 \mathrm{~km}, R_{1}=900 \mathrm{~km}$.

This idealized flow (Fig. 2) consists of two highs (anticyclones) and two lows (cyclones) and an east-west confluence zone exists between the high-low couplets to the north and the lowhigh couplets to the south. In the confluence zone, both vorticity and divergence are actually small or zero while deformation is large (Fig. 3). The value of deformation is $3.2 \times 10^{-5}$ in the middle part of the domain (region with orange shading in Fig. 3a) while both divergence and vorticity are zero in this region (Fig. 3b and 3c). Note that the two rings of blue and orange in the divergence field are the results of the truncation errors in the finite difference calculation and the discontinuity between the regions where the flows are defined by different functions. The same reason leads to the two rings in vorticity and deformation fields.

For typical Meiyu frontal systems, the horizontal temperature gradient is usually weak, therefore the classical frontogensis processes associated with deformation flow is less important. We assume a uniform background potential temperature and an initial background relative moisture field that is only a function of $y$, and is specified as $R H=0.9-0.008(y-1)$, where $y$ is the grid index in $y$ direction. Subjecting to the advection of this flow, the isolines of relative humidity after 6 hours are concentrated distinctly towards the confluence region of 'saddle' field, while the isolines after $12 \mathrm{~h}$ become even denser (Fig. 4b). After 36 hours, the isolines become much more concentrated (Fig. 4d), indicating a buildup of strong moisture gradient along the confluence zone. In the case of three dimensions, vertical motion along the confluence zone will cause the upward transport of the moisture brought in by the horizontal deformation flow.

Often, the moisture gradient zone is not parallel to the confluence zone. An example of such is when the initial relative humidity is specified by $R H=0.9+0.008(x-y)$, where $x$ is the grid index in the $x$ direction. Fig. 5 shows the evolution of this humidity field subjecting to the advection of the same idealized flow. The contours of relative humidity also become more concentrated as in the previous case; moreover, the orientations of these contours become more and more parallel to the confluence zone (Fig. 5). Eventually, the zone of strong moisture gradient becomes parallel to the confluence zone.

In the real atmosphere, this type of 'saddle' flow is often found. Moreover, it is often associated with precipitation. Since the vorticity and divergence are small in such a flow while the deformation is large, even in the absence of distinct temperature gradient, heavy precipitation can result from the confluence associated with the deformation, through the focusing effect on moisture. The richness of moisture in the confluence zone provides a favorable condition for moist convection, although the actual triggering of convection is usually provided by something else, such as upper-level lifting by a short-wave trough or weak but still present low-level convergence (Maddox et al., 1978; Hoxit et al., 1978; Caracena et al., 1979; Maddox et al., 1980a,b). These will be discussed in detail for two real precipitation cases in sections 4 and 5 .

\section{The total deformation and its prediction equation}

\subsection{The total deformation}

In Petterssen (1956), it is shown that a two-dimensional velocity field, $(u, v)$, can be approximated by a truncated Taylor series,

$$
u=u_{o}+(D+F) x / 2-(r-q) y / 2
$$




$$
v=v_{o}+(r+q) x / 2+(D-F) y / 2 .
$$

where $u_{0}$ and $v_{0}$ are velocity components at the coordinate origin, and $D=\left(\frac{\partial u}{\partial x}+\frac{\partial v}{\partial y}\right), q$ $=\left(\frac{\partial v}{\partial x}-\frac{\partial u}{\partial y}\right), \quad F=\left(\frac{\partial u}{\partial x}-\frac{\partial v}{\partial y}\right)$, and $r=\left(\frac{\partial v}{\partial x}+\frac{\partial u}{\partial y}\right)$, which are, respectively, the divergence, vorticity, stretching deformation and shearing deformation. The equation shows that a $2 \mathrm{D}$ flow can be locally expressed as a linear combination of the above four quantities.

Total deformation is usually defined as $E=\sqrt{F^{2}+r^{2}}$ (Petterssen, 1956; Keyser et al., 1986,1988; Norbury, 2002). We show in the following that the magnitude of total deformation is independent of the coordinate used, the same as the divergence and vorticity.

We rotate the $(x, y)$ coordinate system anticlockwise by angle $\theta$, into a new coordinate system $\left(x^{\prime}, y^{\prime}\right)$. The coordinate transform relations are $x^{\prime}=x \cos \theta+y \sin \theta$ and $y^{\prime}=y \cos \theta-x \sin \theta$ and the relations for inverse transform are $x=x^{\prime} \cos \theta-y^{\prime} \sin \theta$ and $y=x^{\prime} \sin \theta+y^{\prime} \cos \theta$. Therefore $u=u^{\prime} \cos \theta-v^{\prime} \sin \theta$ and $v=u^{\prime} \sin \theta+v^{\prime} \cos \theta$. It can be derived that

$$
\left(\begin{array}{l}
\frac{\partial u}{\partial x} \\
\frac{\partial v}{\partial y}
\end{array}\right)=\left(\begin{array}{l}
\left(\frac{\partial u^{\prime}}{\partial x^{\prime}} \cdot \cos \theta-\frac{\partial v^{\prime}}{\partial x^{\prime}} \sin \theta\right) \cos \theta+\left(\frac{\partial u^{\prime}}{\partial y^{\prime}} \cdot \cos \theta-\frac{\partial v^{\prime}}{\partial y^{\prime}} \sin \theta\right) \cdot(-\sin \theta) \\
\left(\frac{\partial u^{\prime}}{\partial x^{\prime}} \cdot \sin \theta+\frac{\partial v^{\prime}}{\partial x^{\prime}} \cos \theta\right) \sin \theta+\left(\frac{\partial u^{\prime}}{\partial y^{\prime}} \cdot \sin \theta+\frac{\partial v^{\prime}}{\partial y^{\prime}} \cos \theta\right) \cdot \cos \theta
\end{array}\right) \cdot
$$

It can be shown that

$$
\begin{aligned}
& F=\frac{\partial u}{\partial x}-\frac{\partial v}{\partial y}=\left(\frac{\partial u^{\prime}}{\partial x^{\prime}}-\frac{\partial v^{\prime}}{\partial y^{\prime}}\right) \cos 2 \theta-\left(\frac{\partial v^{\prime}}{\partial x^{\prime}}+\frac{\partial u^{\prime}}{\partial y^{\prime}}\right) \sin 2 \theta=F^{\prime} \cos (2 \theta)-r^{\prime} \sin (2 \theta) \\
& r=\frac{\partial v}{\partial x}+\frac{\partial u}{\partial y}=\left(\frac{\partial u^{\prime}}{\partial x^{\prime}}-\frac{\partial v^{\prime}}{\partial y^{\prime}}\right) \sin 2 \theta+\left(\frac{\partial v^{\prime}}{\partial x^{\prime}}+\frac{\partial u^{\prime}}{\partial y^{\prime}}\right) \cos 2 \theta=F^{\prime} \sin (2 \theta)+r^{\prime} \cos (2 \theta),
\end{aligned}
$$

therefore, $E^{2}=F^{2}+r^{2}=F^{\prime 2}+r^{\prime 2}$ where prime denotes the quantity in the rotated coordinate. This says that the magnitude of total deformation is independent of the coordinate system. For this reason, the total deformation is a more meaningful quantity to analyze than the individual components of the deformation, and it will be the primary quantity we examine in this paper. In the next subsection, we will derive the total deformation equation.

\subsection{Total deformation equation}

The horizontal equations of motion in the p-coordinate are

$$
\begin{aligned}
& \frac{\partial u}{\partial t}+u \frac{\partial u}{\partial x}+v \frac{\partial u}{\partial y}+\omega \frac{\partial u}{\partial p}-f v=-g \frac{\partial z}{\partial x}+F_{x} \\
& \frac{\partial v}{\partial t}+u \frac{\partial v}{\partial x}+v \frac{\partial v}{\partial y}+\omega \frac{\partial v}{\partial p}+f u=-g \frac{\partial z}{\partial y}+F_{y} .
\end{aligned}
$$


By the operation of $\frac{\partial}{\partial x}(4 a)-\frac{\partial}{\partial y}(4 b)$, we obtain

$$
\frac{\partial F}{\partial t}+\vec{V} \cdot \nabla F+F D+\left(\frac{\partial \omega}{\partial x} \frac{\partial u}{\partial p}-\frac{\partial \omega}{\partial y} \frac{\partial v}{\partial p}\right)-f\left(\frac{\partial v}{\partial x}+\frac{\partial u}{\partial y}\right)-u \frac{\partial f}{\partial y}=-g\left(\frac{\partial^{2} z}{\partial x^{2}}-\frac{\partial^{2} z}{\partial y^{2}}\right)+\left(\frac{\partial F_{x}}{\partial x}-\frac{\partial F_{y}}{\partial y}\right)
$$

By the operation of $\frac{\partial}{\partial y}(4 a)+\frac{\partial}{\partial x}(4 b)$, we obtain

$$
\frac{\partial r}{\partial t}+\vec{V} \cdot \nabla r+r D+\left(\frac{\partial \omega}{\partial y} \frac{\partial u}{\partial p}+\frac{\partial \omega}{\partial x} \frac{\partial v}{\partial p}\right)+f\left(\frac{\partial u}{\partial x}-\frac{\partial v}{\partial y}\right)-v \frac{\partial f}{\partial y}=-2 g \frac{\partial^{2} z}{\partial x \partial y}+\left(\frac{\partial F_{x}}{\partial y}+\frac{\partial F_{y}}{\partial x}\right)
$$

Then by the operation of $\frac{F}{\sqrt{F^{2}+r^{2}}}(5)+\frac{r}{\sqrt{F^{2}+r^{2}}}(6)$, we obtain the equation for total deformation, $E$,

$$
\frac{\partial E}{\partial t}=\text { term } 1+\text { term2+term3+term4+term5+term6, }
$$

where

$$
\begin{aligned}
\text { term1 } & =-\vec{V} \cdot \nabla E \\
\text { term2 } & =-E \nabla_{h} \cdot \vec{V}, \\
\text { term3 } & =\frac{u F+v r}{E} \frac{\partial f}{\partial y}, \\
\text { term4 } & =-\frac{F}{E}\left(g \frac{\partial^{2} z}{\partial x^{2}}-g \frac{\partial^{2} z}{\partial y^{2}}\right)-\frac{r}{E}\left(2 g \frac{\partial^{2} z}{\partial x \partial y}\right), \\
\text { term5 } & =\frac{F}{E}\left(\frac{\partial \omega}{\partial x} \frac{\partial u}{\partial p}-\frac{\partial \omega}{\partial y} \frac{\partial v}{\partial p}\right)+\frac{r}{E}\left(\frac{\partial \omega}{\partial y} \frac{\partial u}{\partial p}+\frac{\partial \omega}{\partial x} \frac{\partial v}{\partial p}\right), \\
\text { term6 } & =\frac{F}{E}\left(\frac{\partial F_{x}}{\partial x}-\frac{\partial F_{y}}{\partial y}\right)+\frac{r}{E}\left(\frac{\partial F_{x}}{\partial y}+\frac{\partial F_{y}}{\partial x}\right) .
\end{aligned}
$$

Here, $\vec{V}$ is the 3D wind vector and $\nabla_{h} \cdot \vec{V}$ is the horizontal divergence and $\omega=d p / d t$ is the vertical velocity in pressure coordinate. From Eq. (7a), we can see that the local change of total deformation is caused by advection (term1), horizontal divergence (term2), the term related to $\beta$-effect (term3), the pressure gradient term (term4), the vertical velocity contributions (term5), and the term related to frictional force and/or turbulence mixing (term6). 


\subsection{The physical meaning of each term}

For the convenience of discussion, we can choose our coordinate so that the shearing deformation is zero. We consider a pure stretching deformation field defined by $u=\alpha x$ and $v=$ $\alpha y$. The flow described by this set of equations in the region close to the coordinate origin is the typical flow pattern associated with a two-high and two-low arrangement (see Fig. 2). For this flow, $\partial u / \partial x>0$ and $\partial v / \partial y<0$, and in the first quadrant $u>0$ and $v<0$.

Term1 in Eq. (7a) represents the advection of total deformation. The sign of this term $(-\vec{V} \cdot \nabla E)$ is determined by the angle between the velocity vector and the gradient of total deformation. When the angle is less than $90^{\circ}$, the projection of $\vec{V}$ on $-\nabla E$ is positive, so term 1 is positive; otherwise, the term is negative. Term2 represents the change in total deformation due to horizontal divergence. For a pure deformation field, $\nabla_{h} \cdot \vec{V}=0$, so this term is zero.

Term3 is a term that is related to the $\beta$-effect or the longitudinal gradient of Coriolis parameter $f$. Its sign is determined by the interaction between $u$ and $\frac{\partial f}{\partial y}$ under the new coordinate (which makes $r=0$ ) system.

Term 4 represents the effect of pressure force. Under the assumption of a pure stretching deformation flow pattern, it can be rewritten as $-\left(g \frac{\partial^{2} z}{\partial x^{2}}-g \frac{\partial^{2} z}{\partial y^{2}}\right)$. Its contribution to the total deformation is determined by the combination of the shear in $x$ and $y$ directions of pressure force ( $x$ and $y$ represent the new coordinate axis under which the shear deformation is zero.).

Term5 combines the horizontal shear of vertical velocity with the vertical shear of horizontal velocity in the new coordinate system. Under above assumption $(r=0)$, term5 can be rewritten as $\frac{\partial \omega}{\partial x} \frac{\partial u}{\partial p}-\frac{\partial \omega}{\partial y} \frac{\partial v}{\partial p}$. Its contribution to the change of total deformation is determined by the interaction of signs of the components.

\section{The analysis of two rainfall cases in July and October, 2003 over China}

The role of vorticity and divergence during the occurrence and development of torrential rain events has been investigated extensively in the literature (e.g., Herbert-Riehl, 1954; Stanley and Michael, 1978a,b; Liebmann et al., 1998; Davidson et al., 1998; David and Anderson, 2001), but the role of total deformation before the occurrence and during the course of heavy rainfall has received much less attention. Bluestein (1977) studied the synoptic-scale deformation related to tropical cloud bands. Significant correlations were found between the cloud bands and the orientation of the axis of dilatation of objectively analyzed non-divergent winds at the lower troposphere. But studies on the correlations between deformation and precipitation are few. For this reason, two heavy rainfall cases associated with east and north China fronts, respectively, are selected to explore the characteristics and role of total deformation before and during the rainfall events. The orientations of the rain bands were nearly east-west and nearly north-south, respectively, in the two cases. 


\subsection{The heavy rainfall event of July 2003}

A heavy rainfall event associated with a Meiyu front occurred in middle and lower reaches of the Yangtze River (MRYR) over China from 00 UTC 4 July to 12 UTC 5 July 2003. The rainband was oriented in the west-southwest to east-northeast direction. The Meiyu front formed before the onset of precipitation and was maintained during the course of precipitation.

Fig. 6a shows that at the $700 \mathrm{hPa}$ level, there is zone of high equivalent potential temperature, $\theta_{e}$, that clearly marks the Meiyu frontal zone that stretches in the northeastsouthwest orientation between $27^{\circ} \mathrm{N}$ and $36^{\circ} \mathrm{N}$. Along this zone, especially near the northern edge of this zone, a line of strong confluence exists that is also a shear zone (Fig. 6b). This confluent shear zone is a result of the large scale flow pattern that consists of a subtropical high located in northwestern Pacific, a mid-to-high latitude low centered to the north at $126^{\circ} \mathrm{E}$ and $48^{\circ} \mathrm{N}$, and a general anticyclonic flow pattern over much of China north of the Yantze River. This flow pattern differs from the classical 'saddle' type deformation pattern for the lack of another low to the southwest and the oversize of the subtropical high. The result of this is the existence of a significant amount of shear or vorticity along the confluence zone. At the $200 \mathrm{hPa}$ level, the precipitation region is dominated by a divergence flow along the northeast edge of South Asia high (not shown). Therefore, the low-level confluence associated with the deformation flow pattern and the upper-level divergence set up a favorable circulation pattern for heavy Meiyu precipitation.

In order to obtain data at higher spatial and temporary resolutions for the above case and to use the data for a diagnostic study, we performed a numerical simulation using the Advanced Research WRF model, initializing the model using the archived $1^{\circ} \times 1^{\circ}$ NCEP real-time global analyses. The Advanced Research WRF (ARW) dynamic core uses compressible, nonhydrostatic equations. It uses the 3rd-order Runge-Kutta time-integration scheme (Wicker and William, 2002). For our simulations, the physics options used include the Ferrier microphysics scheme and Kain-Fritsch cumulus parameterization scheme (Kain and Fritsch, 1990), MRF boundary scheme (Hong and Pan, 1996), Dudhia shortwave radiation (Dudhia, 1989) as well as RRTM longwave radiation scheme (Mlawer et al., 1997). The NCEP analyses at 6-hour intervals are used as the boundary conditions. The center of the 27-km resolution model domain is at $117^{\circ} \mathrm{E}$ and $32^{\circ} \mathrm{N}$ and has $181 \times 161$ points in the horizontal and 31 non-uniformly-spaced terrainfollowing levels in the vertical. The large time step used is $120 \mathrm{~s}$. The 36-hour integration starts at 00 UTC 4 July 2003 and output at 20-minute intervals are saved. Figs. 7a and 7b show the observed and simulated 36-hour cumulative precipitation between 00 UTC 4 July and 12 UTC 5 July 2003. The simulated and observed maximum precipitation centers in MRYR are located, respectively, at $119.5^{\circ} \mathrm{E}$ and $32.5^{\circ} \mathrm{N}$ and at $119{ }^{\circ} \mathrm{E}$ and $32^{\circ} \mathrm{N}$. Other two precipitation centers located at $114^{\circ} \mathrm{E}$ and $30.5^{\circ} \mathrm{N}$ and at $116.5^{\circ} \mathrm{E}$ and $31.5^{\circ} \mathrm{N}$ are also simulated well. Due to lack of observational data over the ocean, the simulated precipitation can not be validated there. We do not have precipitation data over the Korean peninsula either. Otherwise, the general precipitation patterns along the Yangtze River match rather well. Thus, the simulated data will be used for diagnostic analysis.

\subsection{The heavy rainfall event of October 2003}

Another heavy rainfall event occurred in north China between 00 UTC 10 October and 12 UTC 11 October 2003. The rainband extended in a nearly north-south direction. A front, as seen 
from the equivalent potential temperature field at $900 \mathrm{hPa}$, and a confluent shear line in a southwest-northeast orientation (Figs. 8c and 8d), are clearly evident in the lower troposphere before (Figs. 8a and 8b) and during (Figs. 8c and 8d) the course of precipitation. Cyclonic circulations exist to the north and northwest of the confluent line, a high center located to its southeast and another broad anticyclonic circulation located to its northwest. Again, a cyclonic center associated with a standard 'saddle' deformation pattern is missing to the southwest, as with the July case. Similar to the case of July 2003, a 'saddle' flow pattern also existed before the onset of precipitation (Fig. 8c). This is also a typical case of front-shear line precipitation.

The WRF model with the same physics options as used for the earlier case is used again to simulate this rainfall event. The center of the integration domain is at $115^{\circ} \mathrm{E}$ and $36^{\circ} \mathrm{N}$ and the horizontal resolution is $27 \mathrm{~km}$ and the horizontal grid has $181 \times 161$ points. The interval of output data is again 20 minutes. Figs. 9a and 9b show the observed and simulated 36-hour cumulative precipitation between 00 UTC 10 and 12 UTC 11 October 2003. The strongest precipitation centers are located at $116^{\circ} \mathrm{E}$ and $38^{\circ} \mathrm{N}$ for the simulation but at $116^{\circ} \mathrm{E}$ and $37.5^{\circ} \mathrm{N}$ for the observation. The simulated maximum precipitation amount $(>140 \mathrm{~mm})$ is more than the observation $(\sim 120 \mathrm{~mm})$. Although a spurious weaker precipitation maximum appears at $112^{\circ} \mathrm{E}$ and $31^{\circ} \mathrm{N}$ in the simulation, the overall orientation of the rainband and the precipitation north of $33^{\circ} \mathrm{N}$ are simulated well.

\section{The diagnostic analyses of total deformation and its relationship with precipitation}

\subsection{The July 2003 case}

To examine the possible prognostic value of the total deformation field, as compared to the divergence and vorticity fields, we plot in Fig. 10 these three fields at the $700 \mathrm{hPa}$ at $00 \mathrm{UTC}$ 4 July 2003, before the onset of precipitation. It can be seen from the figure that all three fields show large values along a zone that more or less coincide with the band of the later 6-hour precipitation. The magnitude of absolute divergence is the smallest, with the maximum value being around $4 \times 10^{-5} \mathrm{~s}^{-1}$, while that of deformation is the largest, exceeding $1 \times 10^{-4} \mathrm{~s}^{-1}$. The vorticity maximum is about $7 \times 10^{-5} \mathrm{~s}^{-1}$. This shows quantitatively for such typical flow patterns associated with Meiyu fronts that total deformation is a dominant property of the flow, more so than convergence and vorticity. We also know that convergence and vorticity both have important roles to play in precipitation systems, by producing ascent via low-level convergence forcing, and by inducing low-level convergence via Ekman pumping effect, respectively. Pure deformation contains no divergence or vorticity, but when it is the dominant property of the lowlevel flow, its effect can not be overlooked. In this case, it plays an important role in low-level moisture transport, by bringing in moist air from the south side of the main confluence zone. This situation is similar to that of idealized deformation flow and the associated humidity advection considered in section 2 .

Unlike classical cold or warm fronts, the horizontal temperature gradient associated with the Meiyu fronts is not strong - this can be seen from Fig. 11a for the current case. But the gradient of moisture is strong across the front and in the frontal zone (Fig. 11b). The zone of high relatively humidity at the $700 \mathrm{hPa}$ level is a result of the lifting of moisture by the low-level confluent flow which also transports moisture horizontally into the zone, and the horizontal flow is dominated by the deformation. The results of this case shows that the confluence associated 
with the total deformation focuses the moisture into the confluence zone, which provides a favorable condition for moisture convection. The convergence, though weaker relative to deformation, is still the one that triggers the convection via vertical lifting, but the role of deformation in the moisture transport is also important. Since large values of total deformation is present before the onset of precipitation, it is believed to have significant sense to the prognosis of precipitation.

After 00 UTC 4 July 2003, precipitation began to occur and the rainband formed (Fig. 12) within the zone of large total deformation (Fig. 13). During the course of precipitation, the total deformation maximum is located slightly north of the maximum precipitation. Their trends are consistent with each other and both propagate southward from north of $33^{\circ} \mathrm{N}$ to $30^{\circ} \mathrm{N}$. Two precipitation bands of more than $22 \mathrm{~mm}$ occur between 17 UTC 4 July and 00 UTC 5 July, and between 23 UTC 4 July and 08 UTC 5 July, respectively. A major band of large total deformation extends from $33^{\circ} \mathrm{N}$ at 18 UTC 4 July to $31.5^{\circ} \mathrm{N}$ at 21 UTC 4 July, and there is a slight increase in the total deformation during this period. Comparing Fig. 12 and Fig. 13, one can see that the large value of total deformation proceeds the 6-hour cumulative precipitation maxima by 6 to 12 hours. The 6-hour precipitation reaches its maximum of $30 \mathrm{~mm} / 6 \mathrm{~h}$ at $06 \mathrm{UTC}$ July 5 (Fig. 14a) whereas the 6-hour mean total deformation (Fig. 14b) reaches its peak magnitude of about $1.35 \times 10^{-4} \mathrm{~s}^{-1}$ at 00 UTC 5 July, 6 hour ahead of the precipitation maximum. Above analysis indicates that the total deformation has a predictive value for the precipitation more than 6 hours into the future.

\subsection{The October 2003 case}

Similar to the case of July 2003, a 'saddle' flow pattern existed at 00 UTC 10 October, 2003 (Fig. 8c), before the onset of precipitation. The confluent zone is in a southwest-northeast orientation (Figs. 8c and 8d), with a high or anticyclonic circulation center located to its southeast and another broad anticyclonic circulation located to its northwest. Cyclonic circulations exist to the north and northwest of the confluent line, with the center located to the north of the plotting domain. Again, a cyclonic center associated with a standard 'saddle' deformation pattern is missing to the southwest, as with the July case.

The zone of dense isolines of relative humidity (not shown), the 'saddle' confluence shear zone (Figs. 8c and 8d) and the zone of large total deformation (not shown) all stretch in the north-northeast to south-southwest orientation and propagated southeastwards gradually during this precipitation event. Furthermore, the maximum value of total deformation is larger than the maximum absolute values of vorticity and divergence (not shown).

Both orientation and location of the zone of large total deformation (not shown) coincided with the confluent shear line (Figs. 8c and 8d) in the lower troposphere, where the precipitation was to occur. The rainband lied a little to the south of these bands and was in the same orientation. Similar to the case of July 2003, during the course of precipitation, the band of large total deformation (Fig. 15) lies slightly to the west of the rain band (Fig. 16). The value of total deformation increases along with the amount of future 6-hour precipitation (Figs. 15 and 16). From 00 UTC 11 to 06 UTC 11 October 2003, the 6-hour accumulative precipitation reaches a peak value of $24 \mathrm{~mm} / 6 \mathrm{~h}$ (Fig. 17a), whereas the 6-hour time-mean of the total deformation (Fig. 17b) reaches its maximum magnitude of about $2.5 \times 10^{-4} \mathrm{~s}^{-1}$ between 18 UTC 10 and 00 UTC 11 October, which is also ahead of the precipitation maximum. This again indicates certain prognostic value of the total deformation for future precipitation. 
Based on the above analyses on two rainfall cases whose rainbands had different orientations, some conclusions are drawn as follows. Before and during the course of precipitation, both the location and stretching directions of the band of large total deformation and of the confluent shear line overlap in the lower troposphere. The rainbands are located slightly to their southwest and the stretching direction of rainband is generally the same as the large-value zone of deformation. The value of total deformation in the lower troposphere increases before the precipitation reaches their maximum intensity, and the time lag of the latter is often more than 6 hours. The magnitudes of both low-level divergence and vorticity are smaller than that of total deformation for the two typical precipitation cases examined, suggesting that the total deformation is at least an as important a precursor of heavy precipitation of the type analyzed here.

\section{The relative contributions of the terms in deformation equation}

In the previous section, we analyzed the low-level deformation fields for two heavy rainfall cases that occurred over China, and discussed their location and orientation relative to the confluence zone and the bands of strong vorticity and divergence. For these cases, the deformation is found to have larger absolute magnitudes than either divergence or vorticity. The importance of deformation field in these systems is evident. Further, in section 3, we derived the total deformation equation. In this section, using the WRF model output, we calculate the right hand side terms of total deformation equation (7a) except for the friction term, for the two rainfall cases, in order to gain a better understanding of the processes contributing to the time evolution of total deformation.

For the July 2003 case, since the largest precipitation appears in the region of $114^{\circ} \mathrm{E}$ $120^{\circ} \mathrm{E}$ and $30^{\circ} \mathrm{N}-33^{\circ} \mathrm{N}$ (Figs. 7a and $7 \mathrm{~b}$ ), this region is chosen to calculate the area-mean of the terms in Eq. (8a) and the time tendency (sum $=$ term $1+$ term $2+\ldots \ldots+$ term5) from 00 UTC 4 July to 12 UTC 5 July 2003. The time series of these terms are plotted in Fig. 18a. To identify the major processes that are responsible for the tendency of total deformation, the linear correlation coefficients and root-mean-squared (RMS) differences between the sum and terms 1 through 5 are calculated and presented in Table 1. It can be seen that term 2 has a negative correlation coefficient with the sum whereas other terms are have positive correlations. Term4 has the largest positive correlation coefficient of 0.979 , and the smallest RMS difference of $5.1 \times 10^{-10} \mathrm{~s}^{-2}$ among all terms. Terms 1 and 5 have moderate positive correlation coefficients about 0.5 and small RMS differences $\left(\sim 2.3-3.0 \times 10^{-8} \mathrm{~s}^{-2}\right)$.

For the rainfall case of October 2003, the largest precipitation appears in the region of $114^{\circ} \mathrm{E}-120^{\circ} \mathrm{E}$ and $36^{\circ} \mathrm{N}-42^{\circ} \mathrm{N}$ (Figs. 9a and 9b), this region is also chosen to calculate the areamean of the terms in Eq. (7a) and the time tendency at the sum of the terms from 00 UTC 10 to 12 UTC 11 October 2003. The time series of these terms are plotted in Fig. 18b and the correlation coefficients and RMS differences between individual terms and the sum are given in Table 2. Similar conclusion is derived. Also term 2/ term 4 have negative correlation/ largest positive correlation with the sum, and the largest/ smallest RMS difference among all terms.

Above analysis indicates that the pressure gradient term is a major contributor to the local change of total deformation whereas the vertical velocity term and the advection term also have considerable contributions. Since the effect of pressure gradient term is dominant, in the deformation-dominant flow pattern where the high-low and low-high respectively couplet to both sides of the confluence zone, the pressure gradient is not only a force to trigger and 
maintain this kind of flow pattern, but it accelerates the deformation flow. Moreover, the focus of mass field towards the confluent zone, caused by the advection of deformation flow, has some positive feedback to the pressure gradient between both sides of confluence zone. Therefore, the pressure gradient force has close relation to the change of local deformation. It is the basic and the dominant factor inducing the change of deformation-dominant flow pattern. Also due to the deformation-dominant flow pattern, the divergence is small. So the term 2 has the smallest contribution to the local change of deformation. It is evident that the advection will change the local deformation. As for the vertical velocity term, as above mentioned in section 2, in the case of three dimensions, vertical motion along the confluence zone will cause the upward transport of the moisture brought in by the horizontal deformation flow. It plays a role of adjusting the mass field and wind field, namely, the pressure and flow fields, therefore leads to the change of local deformation.

\section{Conclusions}

In this paper, it is shown that the total deformation is an invariant independent of the coordinate system it is expressed in. An idealized flow field is constructed to demonstrate the advection effect on 2D moisture fields by an essentially non-divergent and irrotational deformation flow that is similar to the flow patterns associated with typical heavy precipitation systems over eastern China, especially those associated with the Meiyu front. Two heavy rainfall cases are examined to explore the characteristics and role of total deformation before and during the heavy rainfall. Numerical simulations of these two events are performed using the WRF model at a $27 \mathrm{~km}$ horizontal resolution and the model outputs are used for diagnostic analyses on these cases.

It is found that before the onset of precipitation, the confluence effect associated with the deformation and the transport of moisture by such a flow sets up a favorable condition for heavy precipitation. During the precipitation period, both the location and orientation of the band of large total deformation and those of the confluent shear line are almost identical in the lower troposphere. The precipitation bands are usually located slightly to the south of the shear line. The value of total deformation in the lower troposphere increases before and during the precipitation. The total deformation usually reaches its maximum value about 6 hours before the precipitation reaches its peak intensity. Therefore, the study on total deformation may be helpful for studying precipitation events, especially for the events associated with Meiyu front and with deformation-dominant flow patterns.

To identify the major contributors to the change of total deformation, the total deformation equation is derived, in a similar way as the divergence and vorticity equations are. The calculations of individual terms in the equation show that the pressure gradient term is the largest contributor to the local change of total deformation whereas the vertical velocity term and advection terms also contribute to the change.

In this paper, only two heavy rainfall cases are analyzed. To obtain more general conclusions, more cases should be examined, which is planned for the future. Data of higher temporal and spatial resolutions should be used to explore the relationship between the total deformation and the precipitation in more detail.

Acknowledgement. S. Gao and S. Yang were supported by the National Natural Science Foundation of China under the Grants No. 40433007 and 40333028 and by the Olympic Project 
under grant No. KACX1-02. M. Xue acknowledges the support from the Chinese Academy of Sciences (Grant No. 2004-2-7) which enabled the collaboration.

\section{References}

Bluestein, H. B., 1977: Synoptic-Scale Deformation and Tropical Cloud Bands. J. Atmos. Sci., 34, 891-900.

Caracena, F., R.A. Maddox, L. R. Hoxit and C.F. Chappell, 1979: Mesoanalysis of the big Thompson storm. Mon. Wea. Rev., 107, 1-17.

David, J. S., and J. L. Anderson, 2001: Is midlatitude convection an active or passive player in producing global circulation patterns. J. Climate., 14, 2222-2237.

Davidson, N. E., K. Kurihara, T. Kato, G. Mills, and K. Puri, 1998: Dynamics and prediction of a mesoscale extreme rain event in the Baiu front over Kyushu, Japan. Mon. Wea. Rev., 121, 2005-2029.

Davies-Jones, R. P., 1984: The origin of updraft rotation in supercell storms. J. Atmos. Sci., 41, 2991-3006.

Droegemeier, K. K., S.M. Lazarus and R. P. Davies-Jones, 1993: The influence of helicity on numerically simulated convective storms. Mon. Wea. Rev., 121, 2005-2029.

Dudhia, J., 1989: Numerical study of convection observed during the winter monsoon experiment using a mesoscale two-dimensional model. J. Atmos. Sci., 46, 3077-3107.

Etling, D., 1985: Some aspects of helicity in atmosphere flows. Beitr. Phys. Atmos., 58, 88-100.

Harasti, P.R., and R.List, 2005: Principal component analysis of Doppler radar data. Part I: Geometric connections between eigenvectors and the core region of atmosphere vorticies. J. Atmos. Sci., 62, 4027-4042.

Herbert, R., 1954: Rainfall and vorticity advection. J. Atmos. Sci., 5, 425-425.

Hong, S.Y., and H.L. Pan, 1996: Non-local boundary layer vertical diffusion in a medium-range forecast model. Mon. Wea. Rev., 124, 2322-2339.

Hoxit, L.R., J. M. Fritsch and C.F. Chappell. 1978: Reply. Mon. Wea. Rev., 106, 1034-1034.

Kain, J. S., and J. M. Fritsch, 1990: A one-dimensional entraining/detraining plume model and its application in convective parameterization. J. Atmos. Sci., 47, 2784-2802.

Keyser, D., M. J. Pecnick and M. A. Shapiro, 1986: Diagnosis of the role of vertical deformation in a two-dimensional primitive equation model of upper-level frontogenesis. J. Atmos. Sci., 43, 839-850.

Keyser, D., J. M. Reeder and J. R. Reed, 1988: A generalization of Petterssen's frontogenesis function and its relation to the forcing of vertical motion. Mon. Wea. Rev., 116, 762-780.

Liebmann, B., J. A. Marengo, J. D. Glick, V. E. Kousky, I. C. Wainer and O. Massamban, 1998: A comparison of rainfall, outgoing longwave radiation, and divergence over the Amazon Basin. J. Climate, 11, 2892-2909.

Lilly, D. K., 1986a: The structure, energetics and propagation of rotation convective storms. Part I: Energy exchange with the mean flow. J. Atmos. Sci., 43, 113-125.

Lilly, D. K., 1986b: The structure,energetics and propagation of rotation convective storms. Part II: Helicity and storm stability. J. Atmos. Sci., 43, 126-140.

Lu Huijuan, and Gao Shouting, 2003: On the helicity and the helicity equation (in Chinese). Acta. Ameteorologica. Sinica., 61, 684-691.

Maddox, R. A., L. R. Hoxit, C. F. Chappell and F. Caracena, 1978: Comparison of meteorological aspects of the big Thompson and rapid city flash floods. Mon. Wea. Rev., 106, 375-389. 
Maddox, R.A., F. Canova and L.R. Hoxit, 1980a: Meteorological characteristics of flash flood events over the western United States. Mon. Wea. Rev., 108, 1866-1877.

Maddox, R.A., L. R. Hoxit and C. F. Chappell, 1980b: A study of tornadic thunderstorm interactions with thermal boundaries. Mon. Wea. Rev., 108, 322-336.

Mlawer, E. J., S. J. Taubman, and P. D. Brown, 1997: Radiative transfer for inhomogeneous atmosphere: RRTM, a validated correlated-k model for the long-wave. J. Geophys. Res., 102, 633-682.

Norbury, J., 2002: Large-Scale Atmosphere-Ocean Dynamics, Vol I. Cambridge University Press. P2-7.

Petterssen, S., 1956: Weather Analysis and Forecasting, Vol I. Science Press. P32-40.

Rossby, C. G., 1937: On the mutual adjustment of pressure and velocity distribution in certain simple current systems, I. J. Marine. Res., 1, 15-28.

Rossby, C.G., 1938: On the mutual adjustment of pressure and velocity distribution in certain simple current systems, II. J. Marine. Res., 2, 239-263.

Stanley, L.U., and G. Michael, 1978a: The role of surface divergence and vorticity in the life cycle of convective rainfall. Part I: Observaton and Analysis. J. Atmos. Sci., 35, 10471062.

Stanley, L.U., and G. Michael, 1978b: The role of surface divergence and vorticity in the life cycle of convective rainfall. Part II: Descriptive Model. J. Atmos. Sci., 35, 1063-1069.

Walter, F., and A.J. Thorpe, 1979: An Evaluation of Theories of Storm Motion Using Observations of Tropical Convective Systems. Mon. Wea. Rev. 107, 1306-1319.

Weisman, M.L., and J.B. Klemp, 1982: The Dependence of Numerically Simulated Convective Storms on Vertical Wind Shear and Buoyancy. Mon. Wea. Rev., 110, 504-520.

Wicker, L.J., and J. S. William, 2002: Time-splitting methods for elastic models using forward time schemes. Mon. Wea. Rev., 130, 2088-2097.

Wiin-Nielsen, A., 1973: Compendium of Meteorology, Vol I., WMO No.364.

Wu Rongsheng, and Tan Zhemin, 1989: Generalized vorticity and potential vorticity conversation law and application (in Chinese). Acta Meteorologica Sinica , 47, 436-442.

Yeh, T.C., 1957: On the formation of quasi-geostrohpic motion in the atmosphere, J. Met. Soc. Japan., The 75th Anniversary Volume, 130-137.

Yeh, T. C., and M. Li, 1982: On the characteristics of scales of the atmospheric motions, J. Met. Soc. Japan., 60, 16-23.

Zeng Qingcun, 1963a: The effect of original disturbance structure on adaptation and the application of observed wind field (in Chinese). Acta Meteorologica Sinica, 33, 37-50.

Zeng Qingcun, 1963b: The adaptation and development in atmosphere. Volume I. (in Chinese). Acta Meteorologica Sinica, 35, 163-174.

Zeng Qingcun, 1963c: The adaptation and development in atmosphere. Volume II. (in Chinese). Acta Meteorologica Sinica, 35, 281-189. 


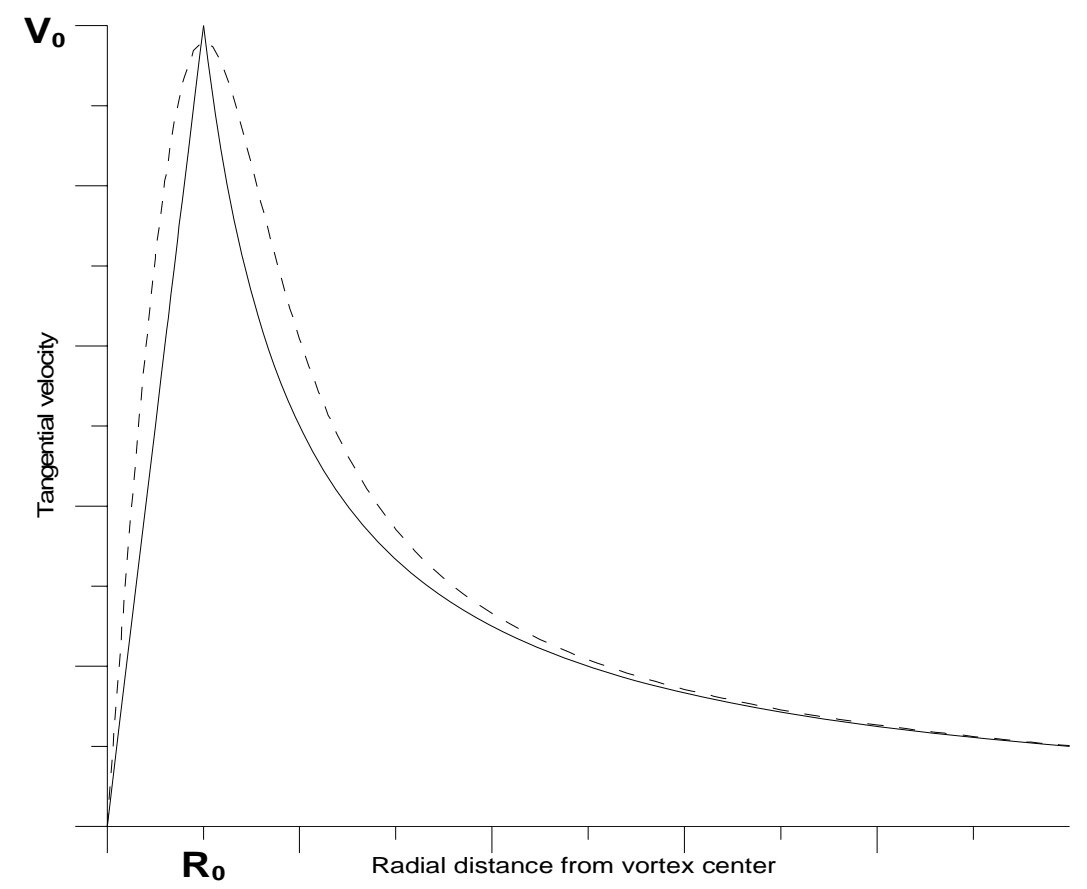

Fig. 1. The tangential velocity derived from traditional Rankine combined vortex (solid line) and vortex (dashed line) looks like a smoothed version of the Rankine combined vortex. The abscissa represents Ridial distance (m) from vortex center, and the ordinate represents the tangential velocity $\left(\mathrm{m} \mathrm{s}^{-1}\right)$.

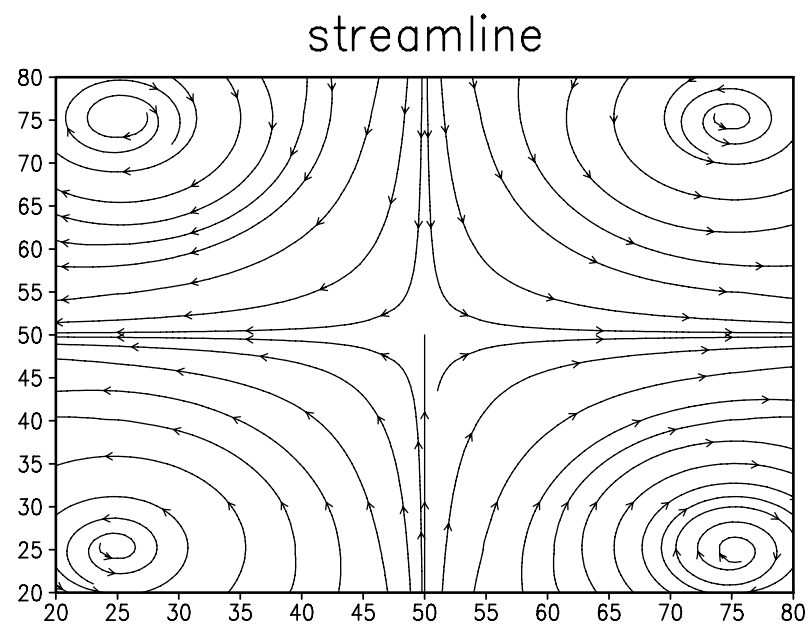

Fig. 2. Constructed idealized flow field with two highs and two lows. The $x$-axis and y-axis denote grid numbers. 

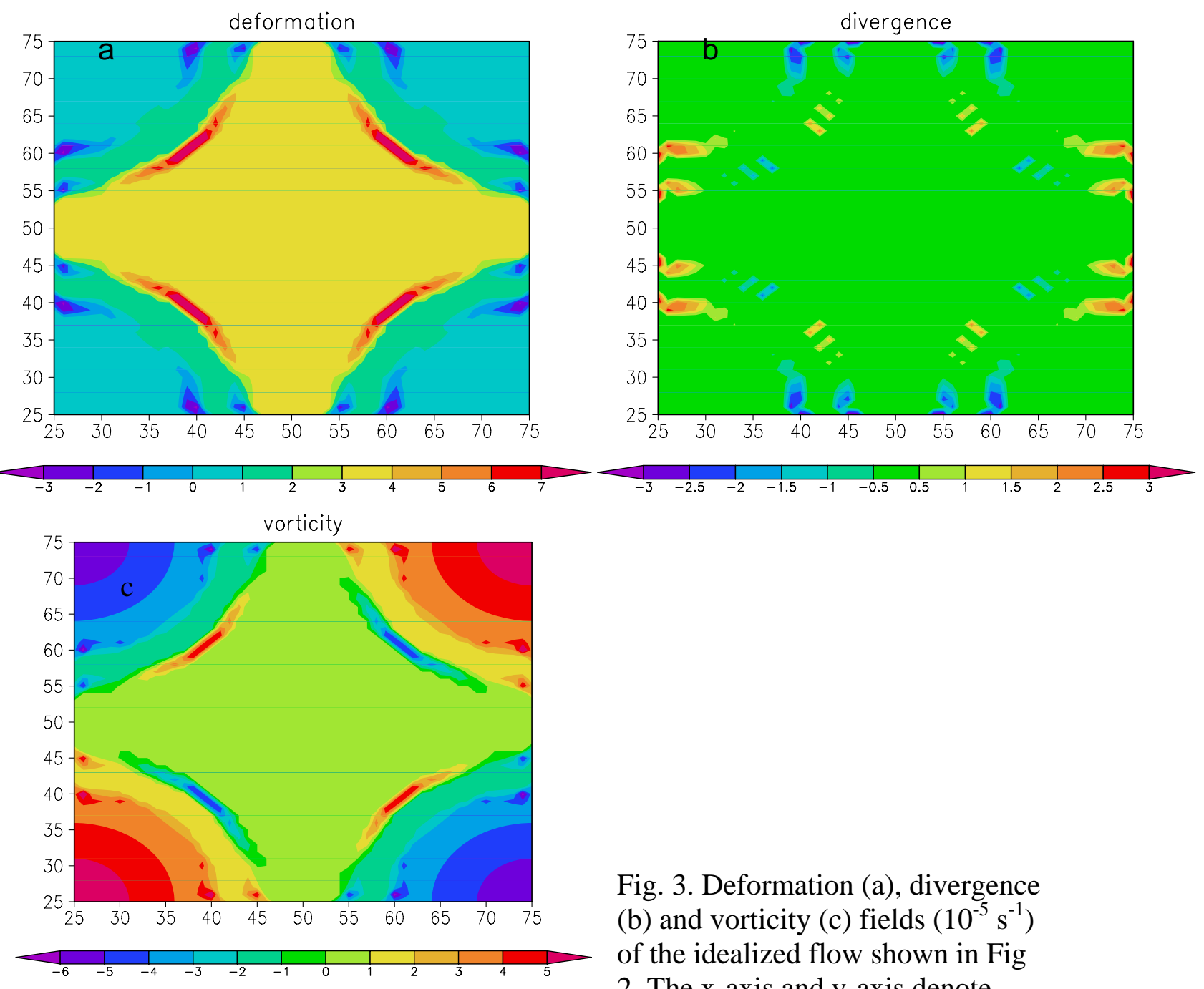

Fig. 3. Deformation (a), divergence (b) and vorticity (c) fields $\left(10^{-5} \mathrm{~s}^{-1}\right)$ of the idealized flow shown in Fig 2. The $x$-axis and $y$-axis denote

grid numbers. 

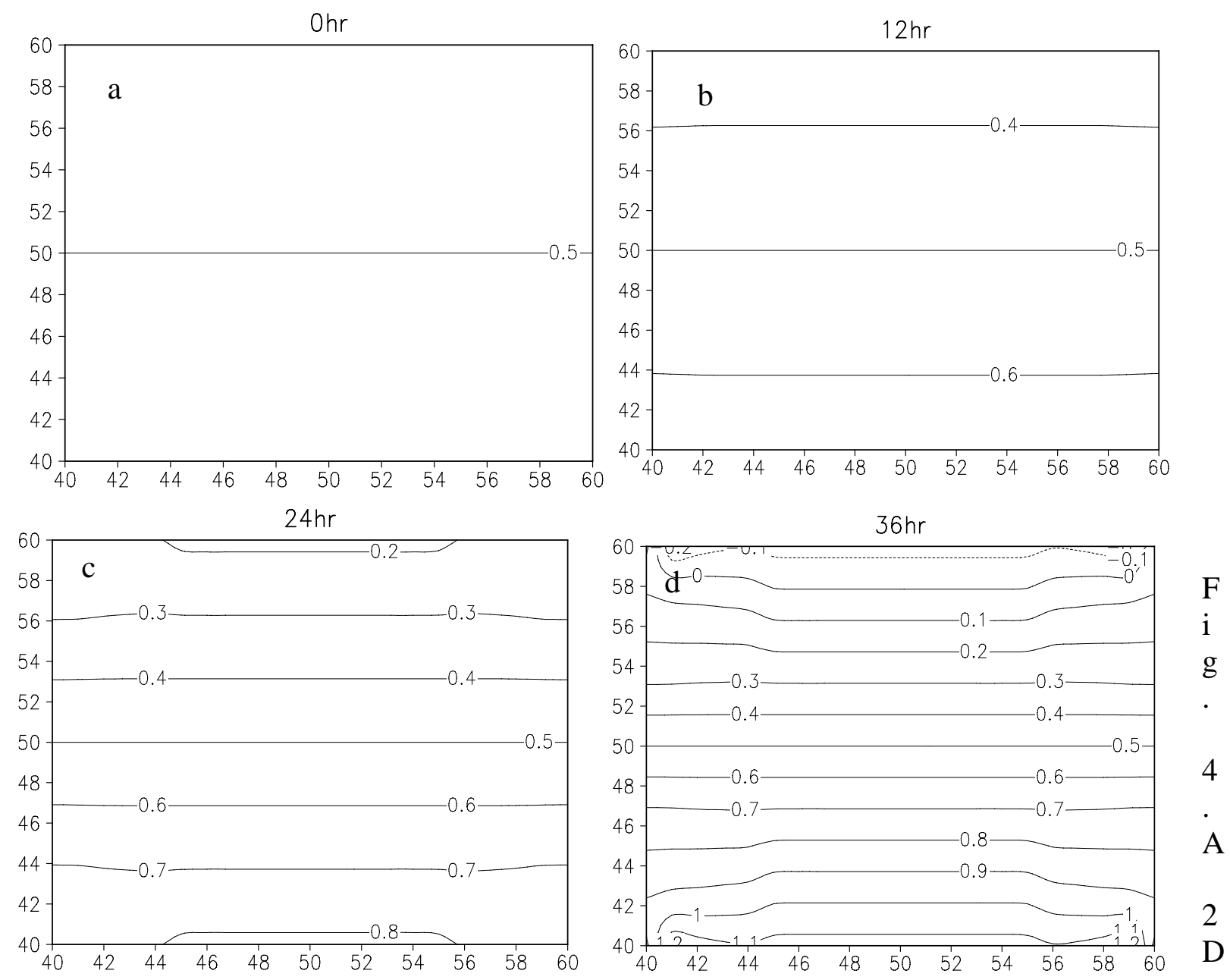

relative humidity field subjecting to the advection by the idealized deformation flow field, at (a) 0 hour, (b) 12 hours, (c) 24 hours and (d) 36 hours. The contour intervals are 0.1, and all contours at time zero have an east-west orientation. The $\mathrm{x}$-axis and $\mathrm{y}$-axis denote grid numbers. 

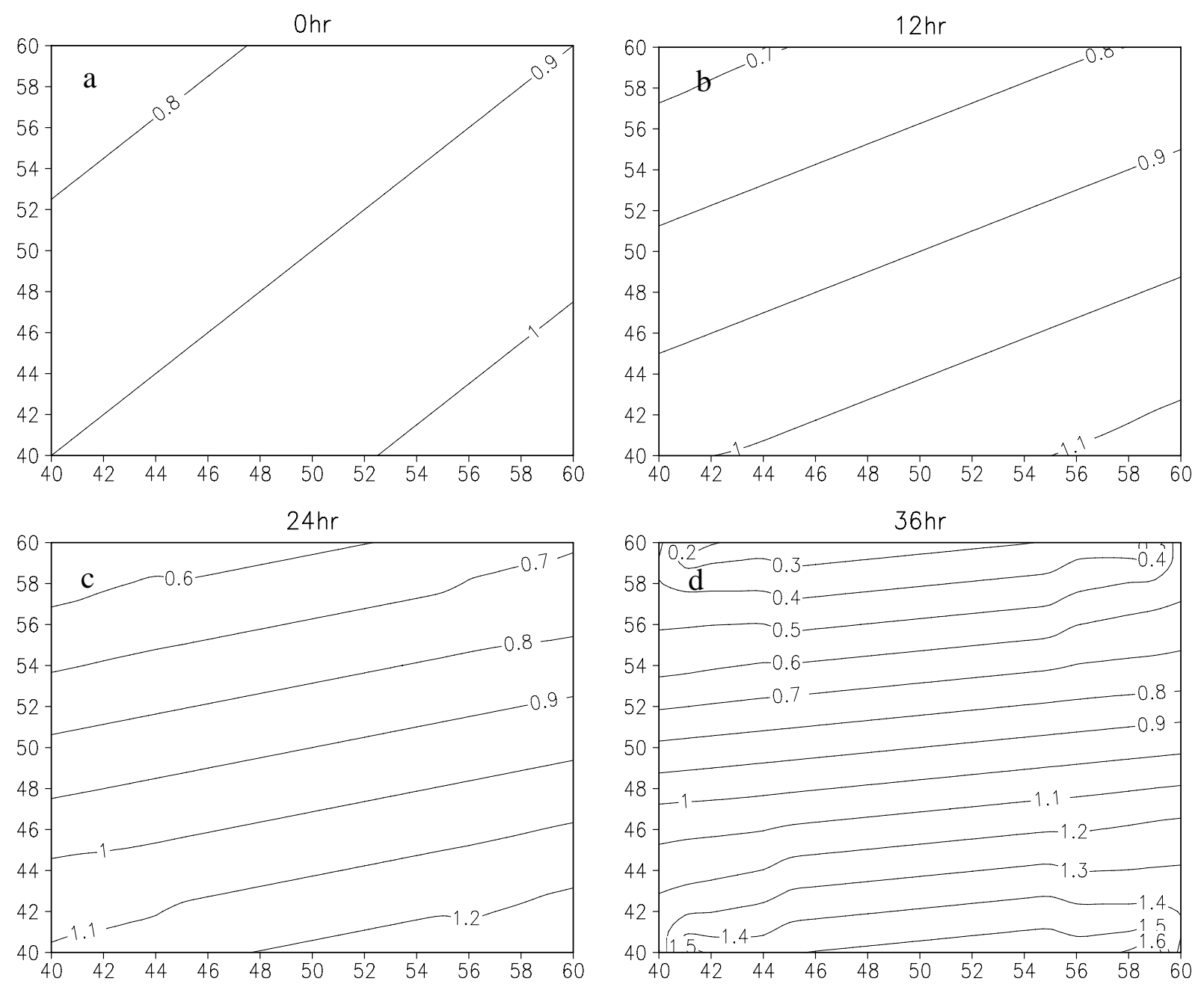

Fig. 5. A 2D relative humidity field subjecting to the advection by the idealized deformation flow field, at (a) 0 hour, (b) 12 hours, (c) after 24 hours and (d) 36 hours. The contour intervals are 0.1 and all contours at time zero have a southwest-northeast orientation. The $\mathrm{x}$-axis and $\mathrm{y}$ axis denote grid numbers. 

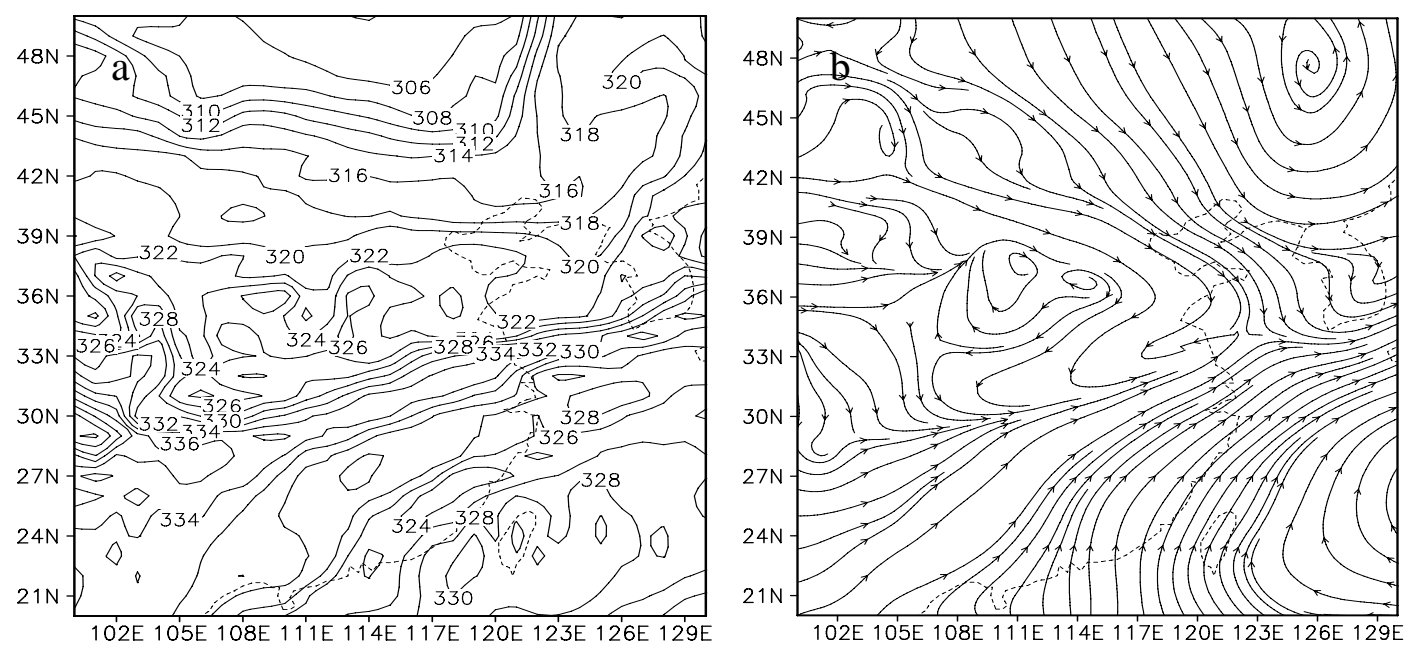

Fig. 6. The (a) equivalent potential temperature (K) and (b) streamline fields at the $700 \mathrm{hPa}$ level at 00 UTC 4 July 2003. 

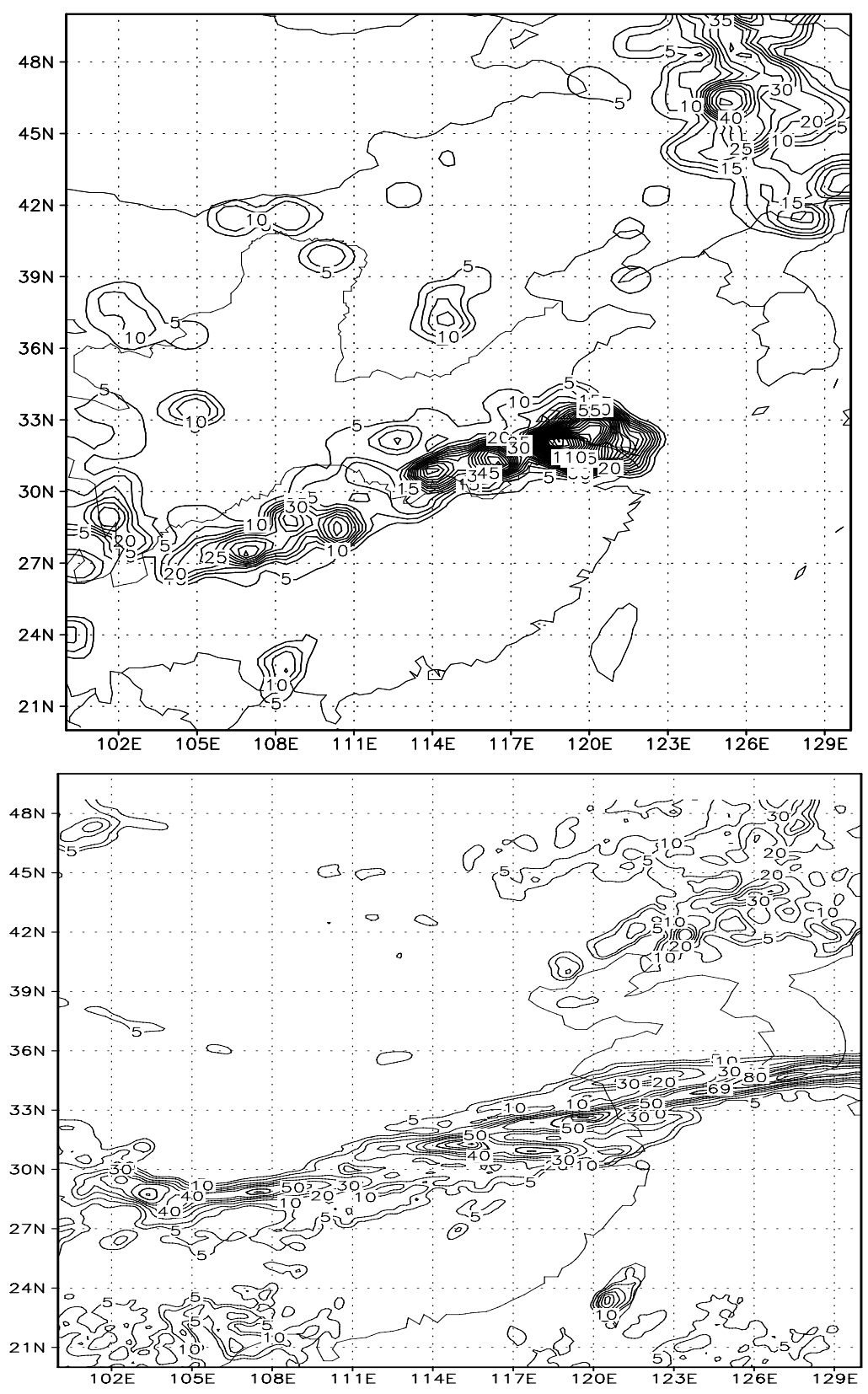

Fig. 7 (a) Observed and (b) simulated 36-hour cumulative rainfall (mm) over the middle and lower reaches of Yangtze River from 00 UTC 4 July to 12 UTC 5 July 2003. 
Fig. 8. The equivalent potential temperature (K) (a, at 00 UTC 10; b, at 00 UTC 11) and streamline (c, at 00 UTC 10; d, at 00 UTC 11
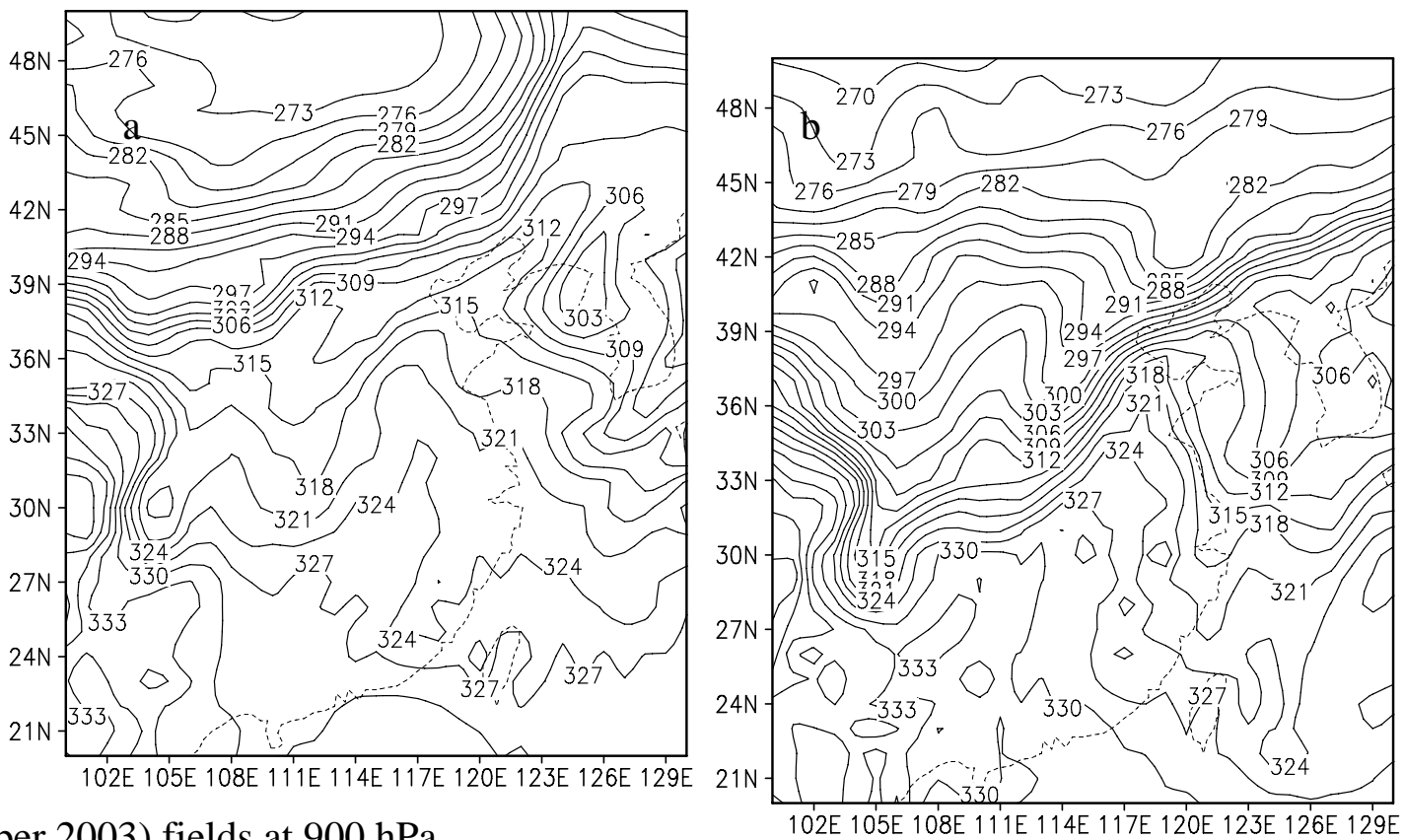

October 2003) fields at $900 \mathrm{hPa}$.
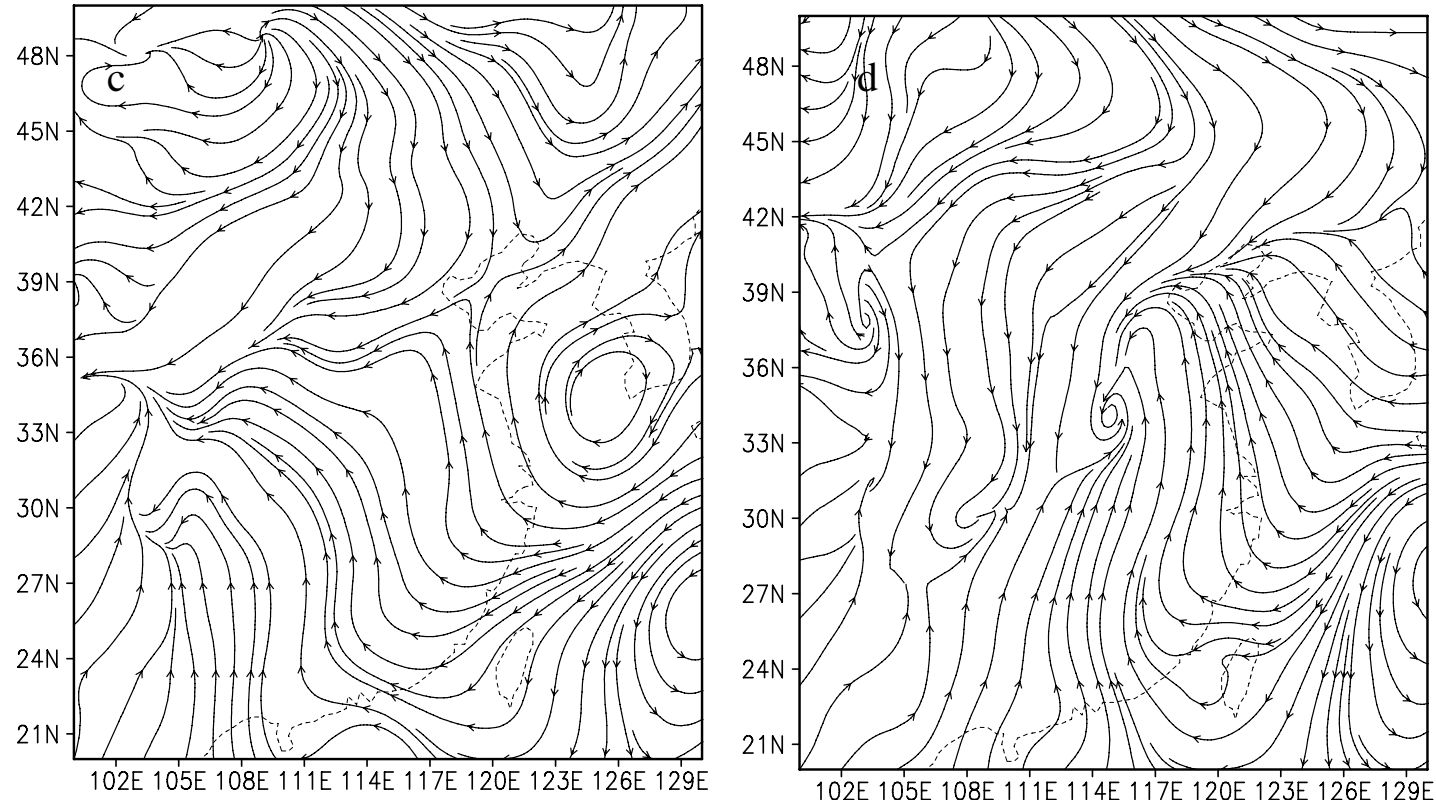

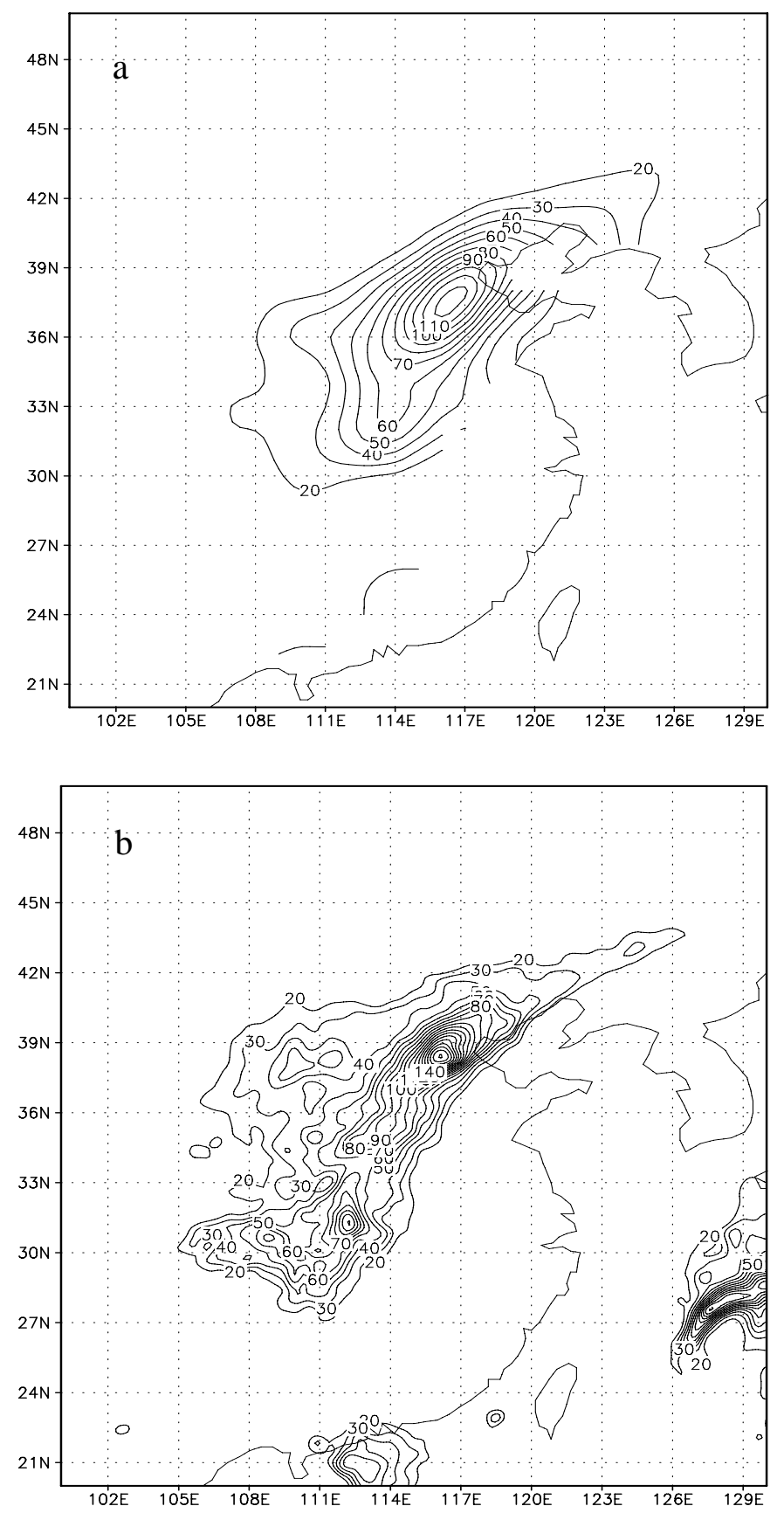

Fig. 9. (a) Observed and (b) simulated 36-hour cumulative rainfall (mm) over the North China from 00 UTC 10 October to 12 UTC 11 October 2003. 

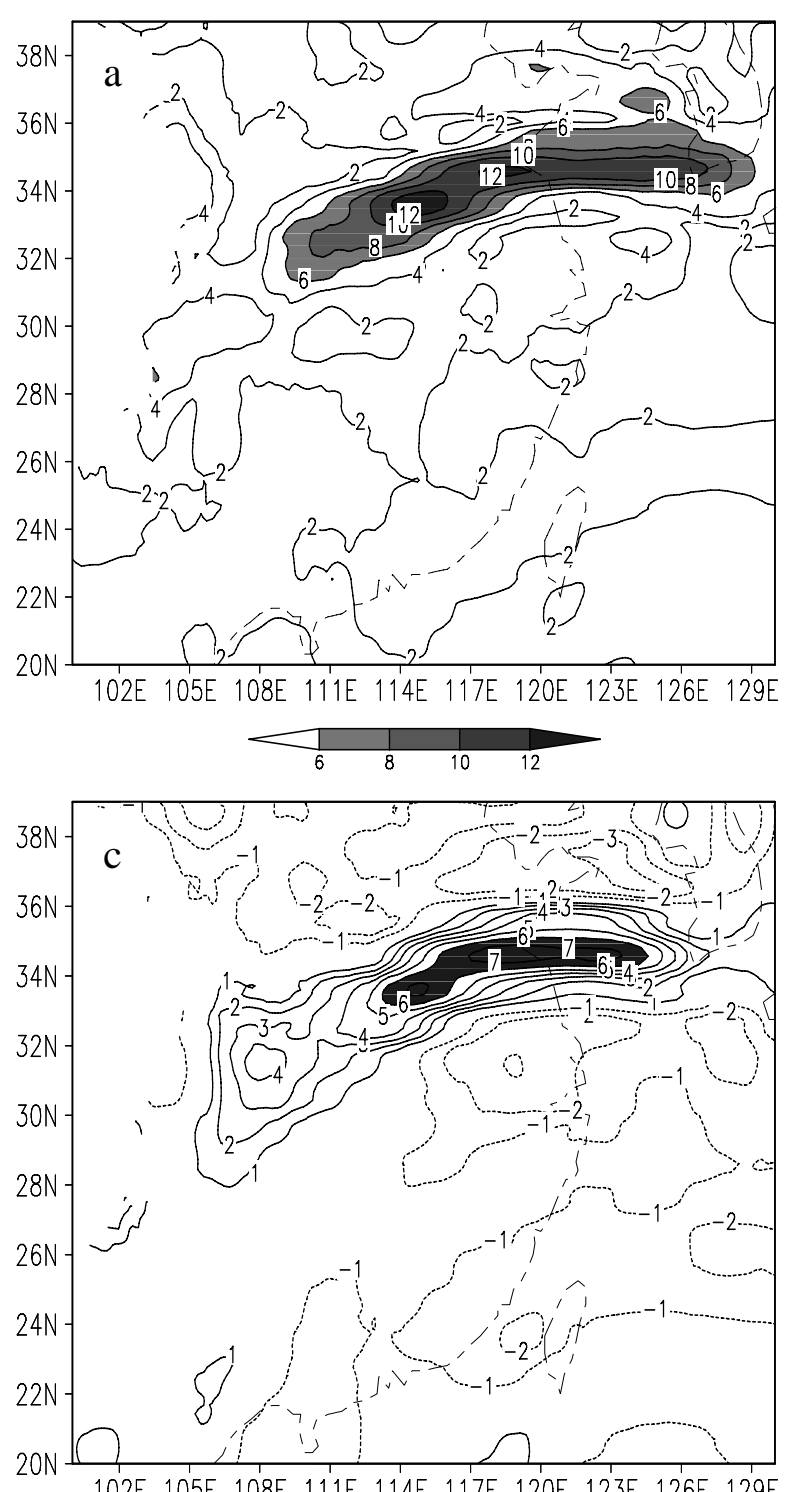

102E 105E 108E 111E 114E 117E $120 \mathrm{E}$ 123E $126 \mathrm{E}$ 129E

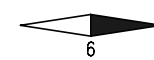

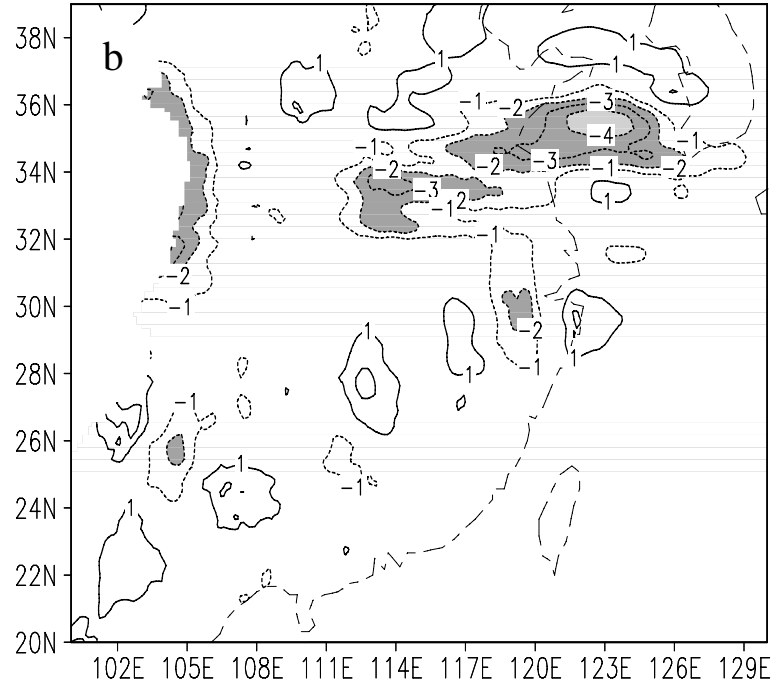

Fig. 10. Total deformation (b) divergence (c) vorticity $\left(10^{-5} \mathrm{~s}^{-1}\right)$ at $700 \mathrm{hPa}$ at 00 UTC 4 July 2003. The shaded areas represent values exceeding $6,-2$ and 6 , respectively, for the plotted fields. 

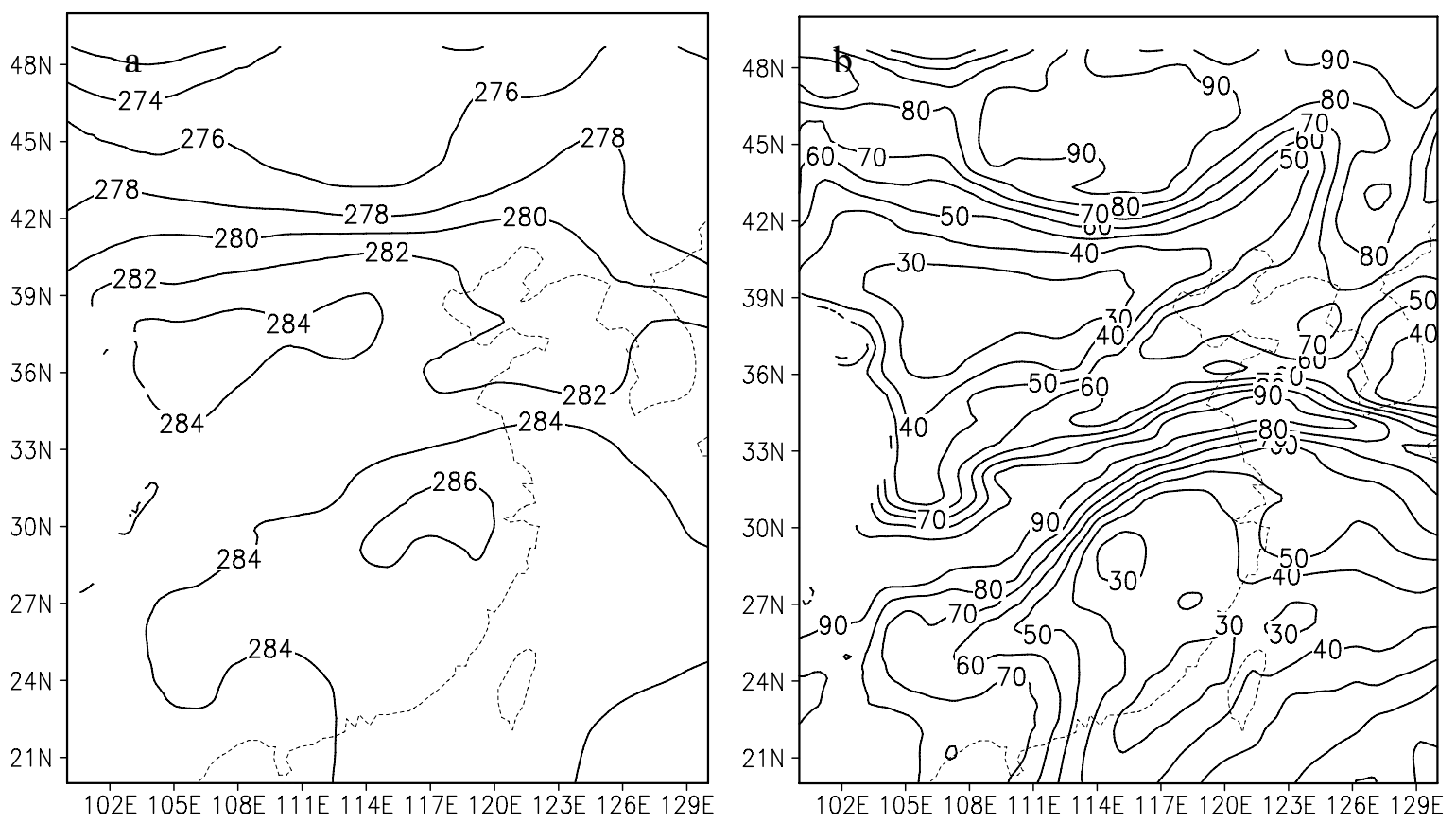

Fig. 11. (a) Temperature (K) and (b) relatively humidity (\%) at $700 \mathrm{hPa}$ at 00 UTC 4 July 2003.

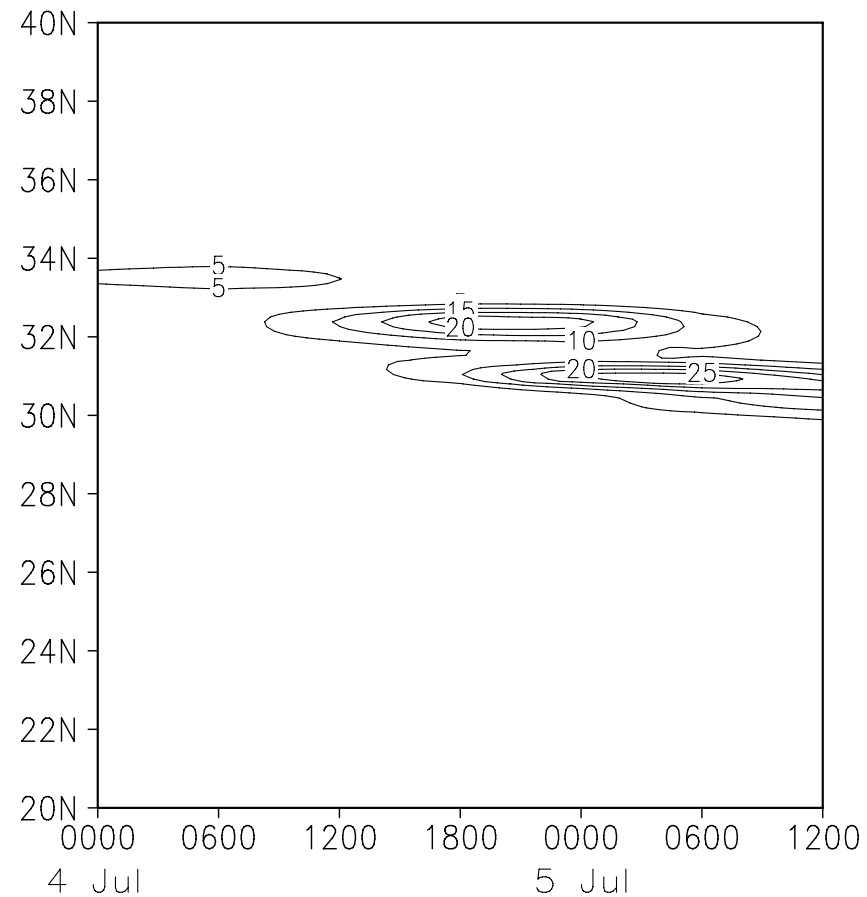

Fig. 12. Time-latitude cross-section of 6-hour cumulative precipitation (mm) along $118^{\circ} \mathrm{E}$ from 00 UTC 4 July to 12 UTC 5 July 2003. The x-axis denotes the date. 


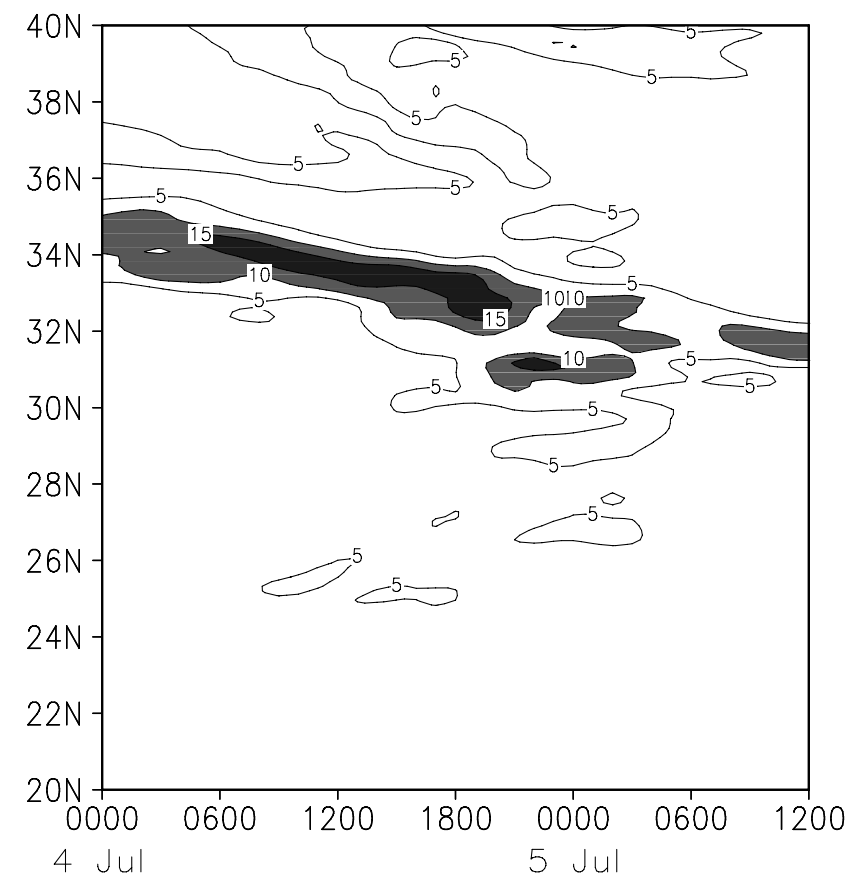

Fig.13. Time-latitude cross-section of total deformation $\left(10^{-5} \mathrm{~s}^{-1}\right)$ along $118^{0} \mathrm{E}$ at $700 \mathrm{hPa}$ from 00 UTC 4 July to 12 UTC 5 July 2003. The regions with total deformation exceeding $1 \times 10^{-4} \mathrm{~s}^{-1}$ are shaded. The x-axis denotes the date. 

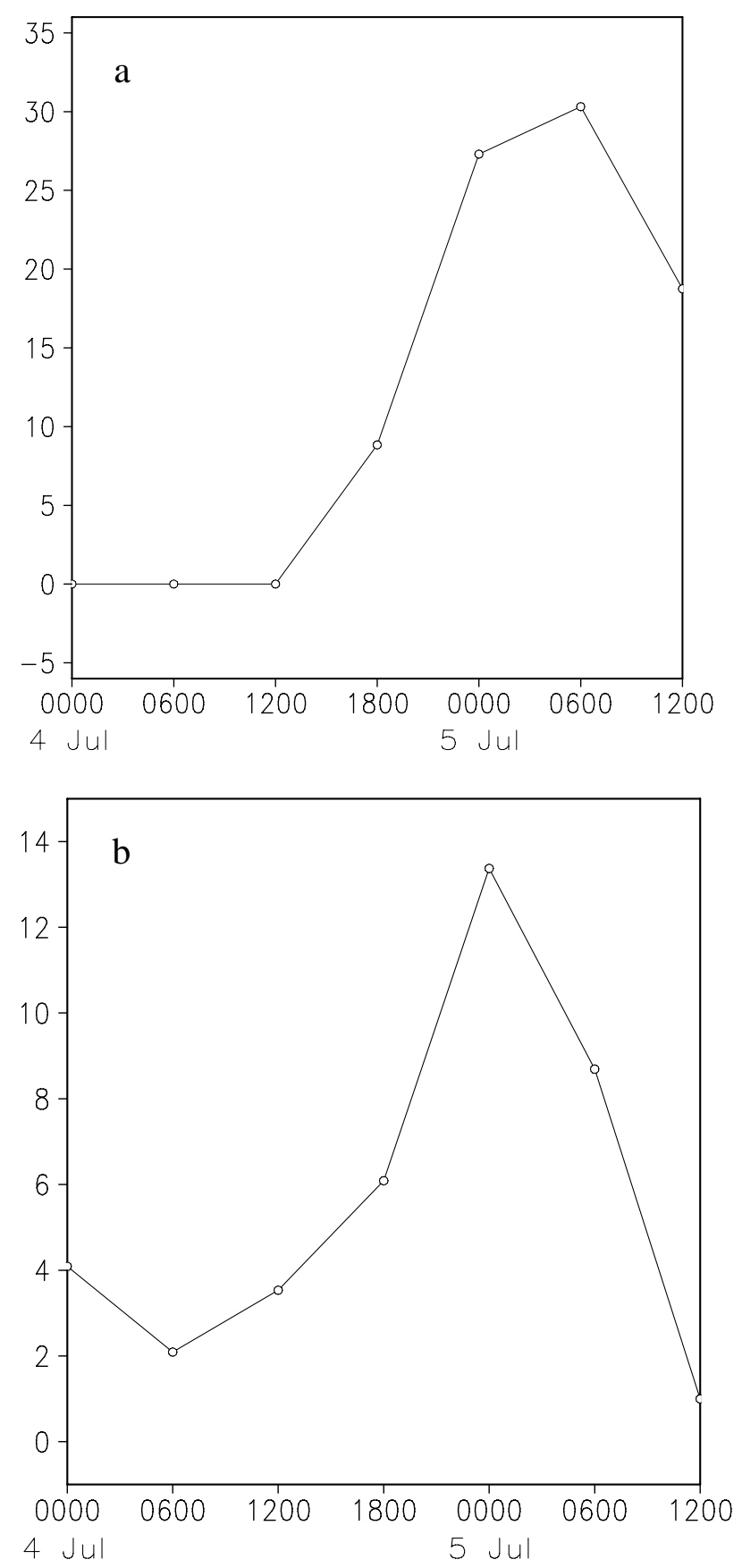

Fig. 14. Time series of (a) 6-hour maximum cumulative precipitation (mm) (b) the magnitude of 6-h time-mean total deformation $\left(10^{-5} \mathrm{~s}^{-1}\right)$ at $700 \mathrm{hPa}$ at the center of precipitation at $119^{\circ} \mathrm{E}, 32^{\circ} \mathrm{N}$ from 00 UTC 4 to 12 UTC 5 July 2003. The x-axis denotes the date. 


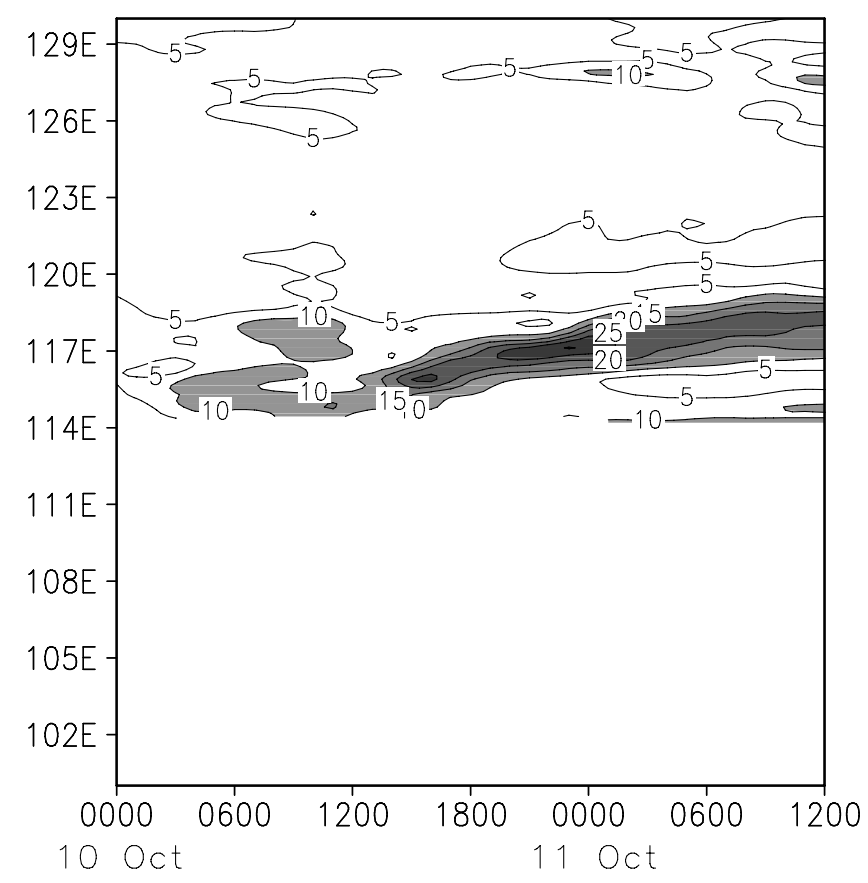

Fig. 15. The time-longitude cross-section along $37^{0} \mathrm{~N}$ of total deformation $\left(10^{-5} \mathrm{~s}^{-1}\right)$ at 900 hPa from 00 UTC 10 October to 12 UTC 11 October 2003. The shaded regions have total deformation values of more than $1 \times 10^{-4} \mathrm{~s}^{-1}$. The $\mathrm{x}$-axis denotes the date. 


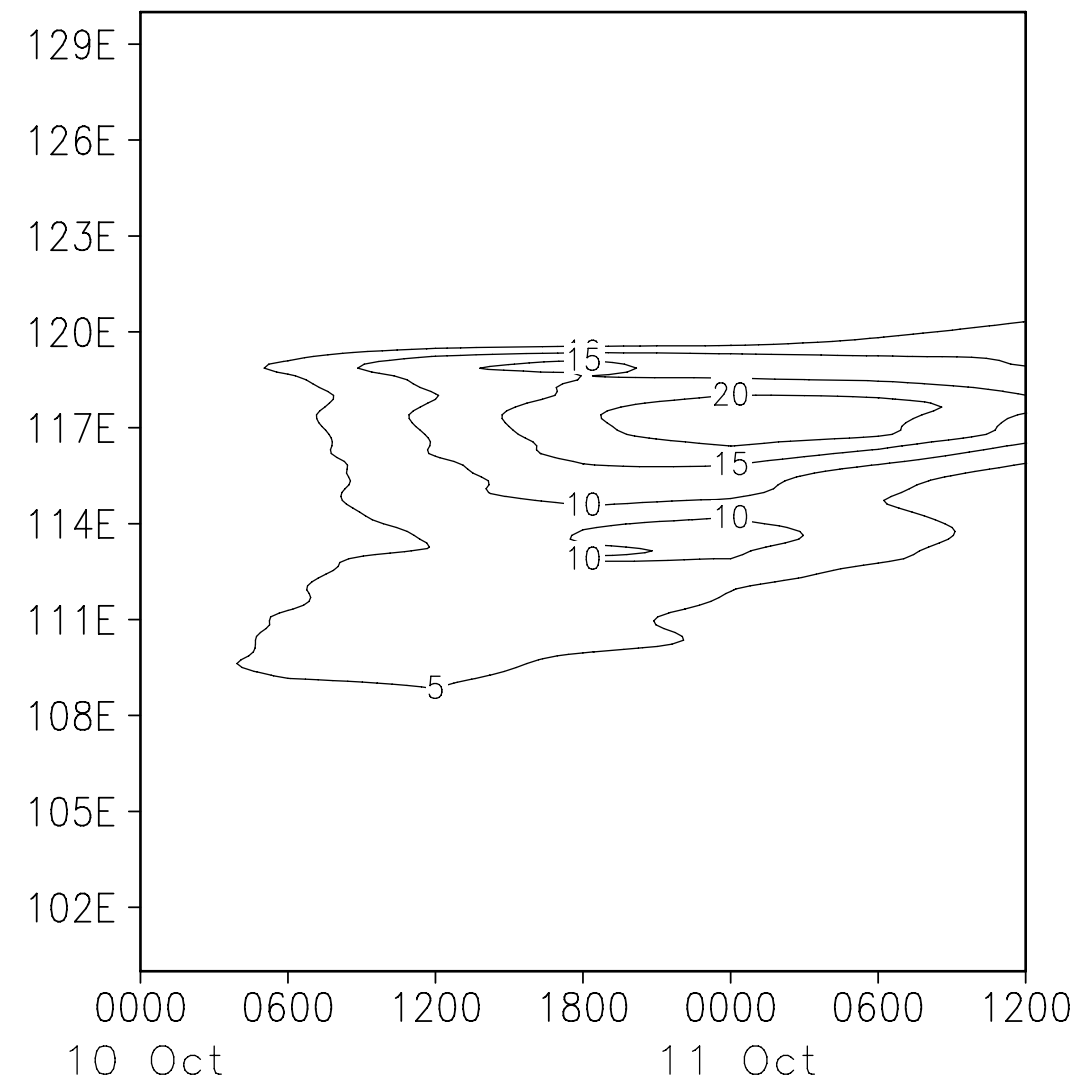

Fig. 16. The time-longitude cross-section along $37^{\circ} \mathrm{N}$ of the 6 -hour cumulative precipitation (mm) from 00 UTC 10 October to 12 UTC 11 October 2003. The x-axis denotes the date. 

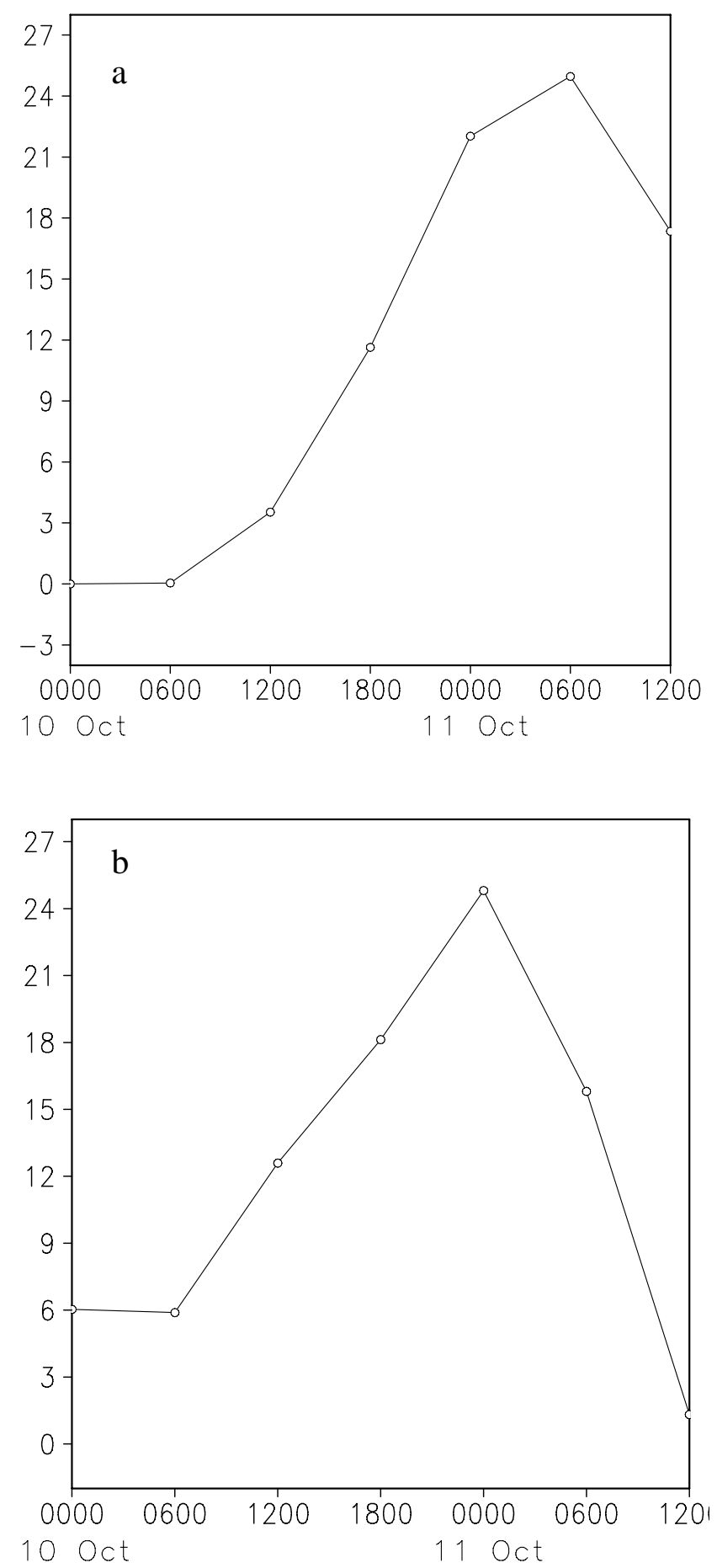

Fig. 17. Time series of (a) 6-hour maximum cumulative precipitation (mm) (b) the magnitude of 6-h time-mean total deformation $\left(10^{-5} \mathrm{~s}^{-1}\right)$ at $900 \mathrm{hPa}$ at the center of precipitation $\left(116^{\circ} \mathrm{E}, 38^{\circ} \mathrm{N}\right)$ from 00 UTC 10 to 12 UTC 11 October 2003. The x-axis denotes the date. 

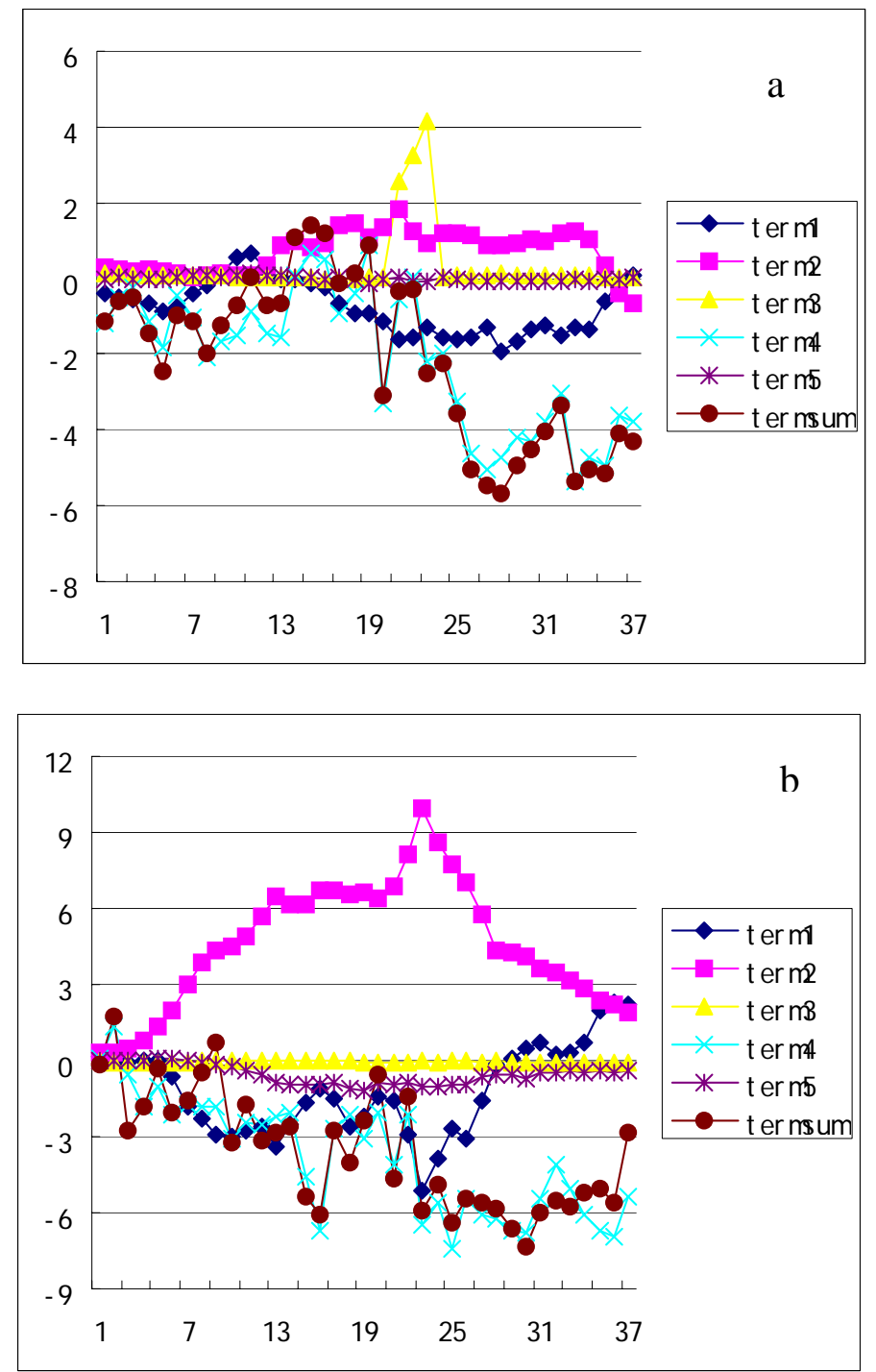

Fig. 18. Time series of area-mean terms 1 though 5 and the sum of these terms in $10^{-9} \mathrm{~kg} \mathrm{~m}^{-3} \mathrm{~s}^{-2}$ for (a) the period from 00 UTC 4 to 12 UTC 5 July 2003 and (b) the period from 00 UTC 10 to 12 UTC 11 October 2003. The abscissa represents time and the ordinate represents the values of terms. The x-axis denotes the time (hour) integrated from (a) 00 UTC 4 July (b)00 UTC 10 October, 2003. 
Table1. The correlation coefficients and RMS differences between individual terms in the total deformation equation and the sum of the terms for the July 2003 case).

\begin{tabular}{|c|c|c|c|c|c|}
\hline Term & 1 & 2 & 3 & 4 & 5 \\
\hline Correlation coefficient & 0.523 & -0.008 & 0.098 & 0.979 & 0.524 \\
\hline $\begin{array}{c}\mathrm{RMS} \\
\left(10^{-8} \mathrm{~kg} \mathrm{~m}^{-3} \mathrm{~s}^{-2}\right)\end{array}$ & 2.976 & 3.581 & 3.309 & 0.051 & 2.310 \\
\hline
\end{tabular}

Table2. The correlation coefficients and RMS differences between individual terms in the total deformation equation and the sum of the terms for the October 2003 case.

\begin{tabular}{|c|c|c|c|c|c|}
\hline Term & 1 & 2 & 3 & 4 & 5 \\
\hline Correlation coefficient & 0.343 & -0.126 & 0.266 & 0.901 & 0.496 \\
\hline $\begin{array}{c}\mathrm{RMS} \\
\left(10^{-8} \mathrm{~kg} \mathrm{~m}^{-3} \mathrm{~s}^{-2}\right)\end{array}$ & 3.864 & 9.028 & 4.200 & 1.046 & 3.691 \\
\hline
\end{tabular}

